ARTICLE

https://doi.org/10.1038/s41467-019-13758-2

\title{
An artificial metalloenzyme biosensor can detect ethylene gas in fruits and Arabidopsis leaves
}

\author{
Kenward Vong (iD) 1,7, Shohei Eda1,2,7, Yasuhiro Kadota (1) ${ }^{3}$, Igor Nasibullin (1) 1, Takanori Wakatake ${ }^{3}$, \\ Satoshi Yokoshima ${ }^{4}$, Ken Shirasu $~^{3}{ }^{3}$ \& Katsunori Tanaka (1) 1,2,5,6*
}

Enzyme biosensors are useful tools that can monitor rapid changes in metabolite levels in real-time. However, current approaches are largely constrained to metabolites within a limited chemical space. With the rising development of artificial metalloenzymes (ArM), a unique opportunity exists to design biosensors from the ground-up for metabolites that are difficult to detect using current technologies. Here we present the design and development of the ArM ethylene probe (AEP), where an albumin scaffold is used to solubilize and protect a quenched ruthenium catalyst. In the presence of the phytohormone ethylene, cross metathesis can occur to produce fluorescence. The probe can be used to detect both exogenous- and endogenous-induced changes to ethylene biosynthesis in fruits and leaves. Overall, this work represents an example of an ArM biosensor, designed specifically for the spatial and temporal detection of a biological metabolite previously not accessible using enzyme biosensors.

\footnotetext{
${ }^{1}$ Biofunctional Synthetic Chemistry Laboratory, RIKEN Cluster for Pioneering Research, 2-1 Hirosawa, Wako-shi, Saitama 351-0198, Japan. ${ }^{2}$ GlycoTargeting Research Laboratory, RIKEN Baton Zone Program, 2-1 Hirosawa, Wako-shi, Saitama 351-0198, Japan. ${ }^{3}$ Plant Immunity Research Group, RIKEN Center for Sustainable Resource Science, 1-7-22 Suehiro-cho, Tsurumi, Yokohama, Kanagawa 230-0045, Japan. ${ }^{4}$ Graduate School of Pharmaceutical Sciences, Nagoya University, Furo-cho, Chikusa-ku, Nagoya 464-8601, Japan. ${ }^{5}$ Biofunctional Chemistry Laboratory, A. Butlerov Institute of Chemistry, Kazan Federal University, 18 Kremlyovskaya street, Kazan 420008, Russia. ${ }^{6}$ Department of Chemical Science and Engineering, School of Materials and Chemical Technology, Tokyo Institute of Technology, 2-12-1 O-okayama, Meguro-ku, Tokyo 152-8552, Japan. ${ }^{7}$ These authors contributed equally: Kenward Vong, Shohei Eda. *email: kotzenori@riken.jp
} 
E nzyme-based chemical biosensors for diagnostic purposes still remains an underdeveloped research area. One of the major issues impeding progress is that most, if not all, enzyme biosensors are derived from existing natural enzymes, rather than methodically designed. As such, there is still a large chemical space that remains inaccessible based on current developmental approaches to enzyme biosensors.

One challenging metabolite to detect is ethylene (IUPAC name: ethene), a well-characterized phytohormone that plays a major role in regulating many aspects of plant growth, immunity, development, and senescence ${ }^{1,2}$. In literature, two systems are known to control ethylene biosynthesis in plants ${ }^{3}$. System 1 production, which is ethylene autoinhibitory ${ }^{4}$, is applicable to all plants and is mainly responsible for controlling basal ethylene levels for normal vegetative growth. Under environmental or developmental stimuli, ethylene levels in climacteric fruit can dramatically increase via system 2 production, which is an ethylene autocatalytic process ${ }^{5-7}$. In literature, exogenous sources of ethylene have been shown to greatly accelerate the processes of abscission and ripening ${ }^{8-11}$.

Given its biological importance, improved diagnostic tools for ethylene gas would be invaluable to agricultural research to help better study ethylene-dependent pathways during stress response, hormone treatment, and different developmental stages in plants. However, since ethylene is a small gaseous olefin that freely diffuses between membranes under physiological conditions, there are many obstacles to overcome in order to develop working systems. Current methods to assay ethylene in plants have largely employed analytical techniques (such as gas chromatography, electrochemical sensors, and laser-based techniques ${ }^{12}$ ), or have utilized plants genetically encoded with ethylene-inducible reporters (such as EBS:GUS ${ }^{13,14}$ ). As an alternative method for ethylene detection, interest has recently begun to shift towards developing chemical-based probes. Through this approach, topical application onto plant tissues should in theory allow ethylene levels to be monitored in a spatiotemporal manner.

In one of the earliest examples of a chemical-based ethylene probe, the Burstyn group developed poly(vinyl phenyl ketone) (PVPK) polymer films. In the presence of ethylene, coordination with impregnated silver ions could lead to the proportional decrease of photoluminescence ${ }^{15}$. Later, the Swager group developed an alternative system where the quenched fluorescence of conjugated polymers by copper(I) scorpionate complexes could be revived by displacement with ethylene ${ }^{16}$. Another notable example is from the Kodera group, where a silver(I)-anthracene complex was constructed around a crucial metal-arene interaction. Upon exposure to ethylene, interconversion from a metal-arene to metal-ethylene interaction led to a change in the anthracene absorption and fluorescence spectra ${ }^{17}$. Finally, during the preparation of this manuscript, two reports were published highlighting an elegant strategy using profluorescent ruthenium metal complexes ${ }^{18,19}$. In the presence of ethylene, turn-on fluorescence can be triggered via cross metathesis to release a BODIPY-based dye. In particular, the Michel group showed the detection of diffused ethylene from live cell cultures incubated with ripening fruit ${ }^{18}$. Although a clear upgrade in terms of biocompatibility compared to previous strategies, the direct imaging of fruits or plants was not shown in this report.

Among all current examples of ethylene-sensing probes, one common theme is the use of water-insoluble metal catalysts. For instance, besides the Michel report, all studies have heavily relied on the use of organic solvent. Furthermore, none of these methods have been able to test ethylene directly from plant tissue; instead relying on exogenous ethylene sources. It can be surmised that in the presence of water-based plant samples, metal complexes may aggregate, potentially lowering their reactivity. In addition, metal quenching in complex biological environments is also an issue. Numerous reports in literature have identified high concentrations of glutathione in plants ${ }^{20}$. As a result, for metals like ruthenium, inactivation by low molecular weight thiols becomes an added challenge. In order to overcome these practical limitations, what is currently lacking is a means to confer biocompatibility to metal complex catalysts.

Artificial metalloenzymes (ArM) are the result of incorporating abiotic metals complexes into a protein scaffold, which can often impart enhanced biofunctionality to the anchored metal catalysts. In current literature, a host of different protein scaffolds have been adapted as ArMs, some of which include streptavidin $^{21-26}$, prolyl oligopeptidase $\mathrm{e}^{27,28}$, myoglobin ${ }^{29,30}$, $\mathrm{CeuE}^{31}$, ADORA2A $\mathrm{A}^{32}$, nitrobindin ${ }^{33}$, among many others. Recently, our group explored the use of human serum albuminbased ArMs, which was found to impart two important biological effects for the anchored metal catalyst ${ }^{34}$. First, the typically insoluble ruthenium complex catalyst (linked to a coumarinligand anchor) can become soluble in aqueous conditions with the addition of albumin. This is likely the effect of the hydrophobic-binding pocket on albumin, which is well known to bind to many hydrophobic drug molecules ${ }^{35}$. And second, due to the negatively charged surface of albumin at physiological $\mathrm{pH}^{36}$, charged metabolites were found to be prevented from entering the hydrophobic-binding pocket. As such, metal catalysts (e.g. ruthenium) bound inside the hydrophobic-binding pocket could be resistant to inactivation from biological thiols (i.e. glutathione). To highlight the biocompatibility of albuminbased ArMs bound with ruthenium (alb-Ru), investigations were then further done to develop an anticancer prodrug therapy based on activation via ring-closing metastasis (RCM) ${ }^{34}$. In this case, alb- $\mathrm{Ru}$ was furnished with cancer cell-targeting complex $N$-glycans ${ }^{37-41}$, which was then used to successfully activate a RCM-drug precursor against various cancer lines (e.g. HeLa, A549, SW620).

Not only restricted to drug therapy, the chemoselective nature of abiotic metal-containing ArMs can also open the door for enzyme biosensor applications. Although metal-based diagnostic probes have been well studied in literature, many of them do not directly engaged with water-based specimens under organic solvent-free conditions. As such, one promising approach is to develop enzyme biosensors based on albumin ArMs to confer water solubility and biocompatibility to metal complexes used for diagnostic purposes.

The principal goal of this paper is to demonstrate that ArMs can be adapted as enzyme biosensors to detect ethylene produced from real world biological samples. The significance of this work is that when compared to current metal-based ethylene probes, it would be one of the first to show applicability with samples directly obtained from fruits and leaves. And when compared to other ethylene detection methods (e.g. GC, electrochemical sensors), it presents a better way to analyze samples in a spatiotemporal manner.

The strategy to develop the ArM ethylene probe (AEP) is outlined in Fig. 1. Using the hydrophobic-binding pocket of albumin, the bound metal complex is composed of three key components: the fluorophore 7-diethylaminocoumarin (DEAC), the second generation Hoveyda-Grubbs catalyst, and the olefincontaining DABCYL quencher. In its normal state, the DABCYL quencher suppresses DEAC fluorescence via Förster resonance energy transfer (FRET) interactions. However, in the presence of ethylene gas, Hoveyda-Grubbs catalyzed cross metathesis will occur, thereby releasing the olefin-containing DABCYL quencher. As a result, detectable levels of fluorescence can be produced. To test this hypothesis, the following study investigates the ability of AEP to detect ethylene in biological samples that include fruits and plant leaves. 


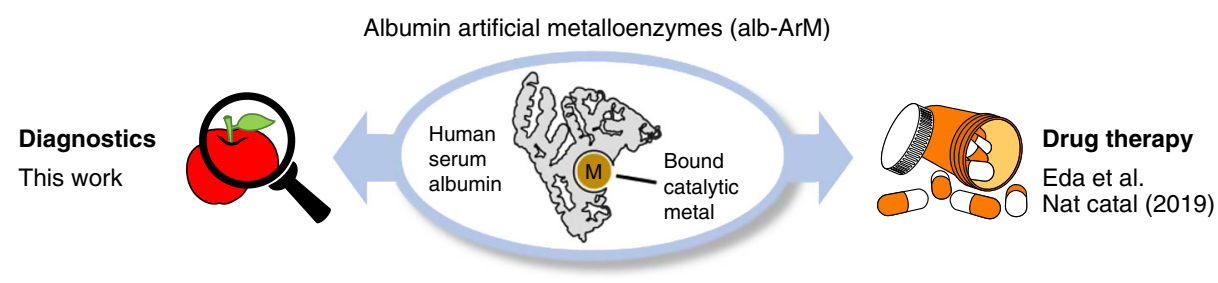

ArM-based ethylene detection in plants:

GOAL

o To create an enzyme biosensor that can detect ethylene in a spatiotemporal manner
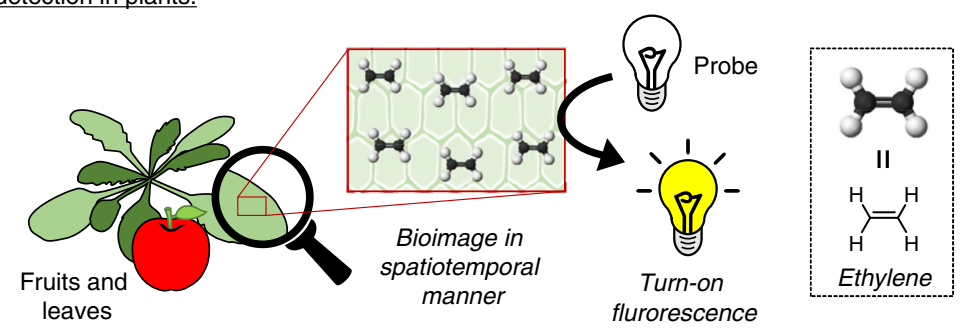

STRATEGY

o Utilize FRET-based detection based on ruthenium-catalyzed, cross metathesis with ethylene gas
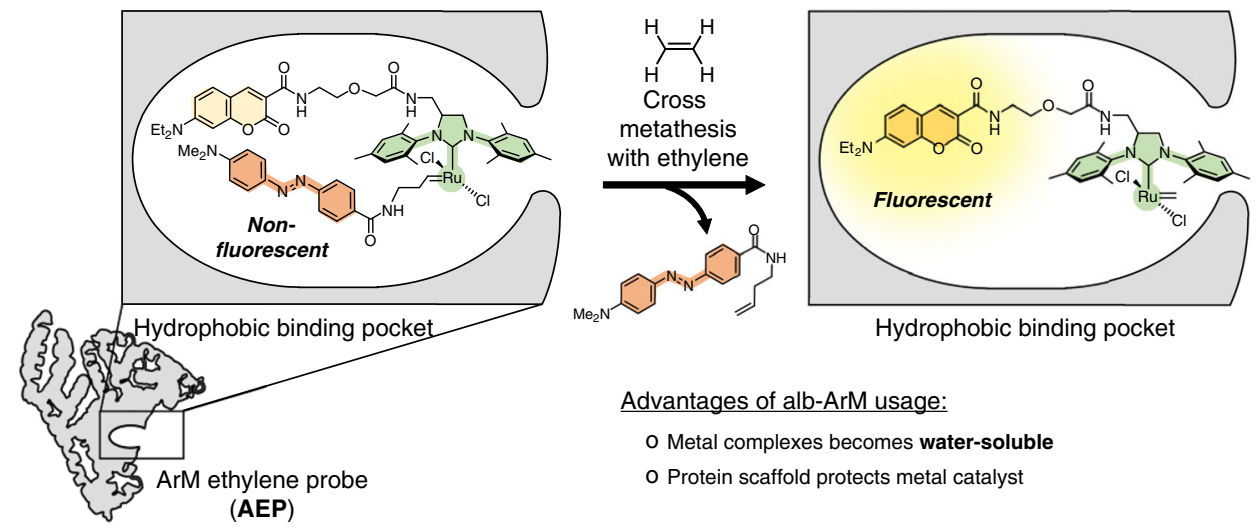

Hydrophobic binding pocket

Advantages of alb-ArM usage:

o Metal complexes becomes water-soluble

o Protein scaffold protects metal catalyst

Fig. 1 The versatility of albumin artificial metalloenzymes allows for its usage in areas such as drug therapy and diagnostics. The goal of this study was to develop a leading example of an ArM-based enzyme biosensor, which can be used to monitor the ethylene produced in fruits and leaves via turn-on fluorescence. The strategy relies on the AEP probe, which uses FRET-based detection of ethylene gas through ruthenium-catalyzed, cross metathesis.

\section{Results}

Preparation of ethylene-sensing ArM. The steps to prepare AEP are outlined in Fig. 2a. Previously in the construction of the albumin ArM (alb-Ru), a DEAC-Ru complex was utilized where a DEAC moiety was used as a ligand anchor to direct the second generation Hoveyda-Grubbs catalyst towards the hydrophobicbinding pocket of albumin. The fluorescence emitted by DEAC itself is highly sensitive to the polarity of the surrounding solvent. As shown in Fig. 2b, the quantum yield of DEAC-Ru increases by roughly 20 -fold in the transition from a polar environment $(10 \%$ dioxane $\left./ \mathrm{H}_{2} \mathrm{O}\right)$ to a non-polar environment $\left(60 \%\right.$ dioxane $\left./ \mathrm{H}_{2} \mathrm{O}\right)$. As such, alb-Ru generally exists as a fluorescent and watersoluble ArM, prepared as described in the "Methods" section.

In literature, DEAC and DABCYL have previously been identified as a FRET donor pair ${ }^{42}$. Therefore, the approach undertaken in this study to prepare the AEP probe is based on reacting alb-Ru with the olefin-containing DABCYL quencher, as described in the "Methods" section. The resulting RuQ complex found inside the albumin-binding pocket exhibits significantly lower levels of fluorescence due to quenching, which is evident when comparing the observed fluorescence between alb-Ru and the AEP probe in Fig. 2c. Furthermore, no major structural changes to the protein complexes were identified during preparation since their circular dichroism (CD) spectra overlapped perfectly (Fig. 2d).
Ethylene-reactivity studies. The theory behind AEP is that biosynthesized ethylene produced by plants will react with the bound ruthenium catalyst, thereby displacing the DABCYL quencher to activate a fluorescent signal. Given the competition with the more reactive ethylene substrate, and the added obstacle of re-entering the albumin-binding pocket under biological conditions, it is surmised that reversible reactivity of the DABCYL quencher will only have a mild influence during bioimaging.

To investigate AEP reactivity, a series of in vitro studies were performed. First, dose-dependent response curves were obtained for RuQ only (Fig. 2e). These experiments were done by injecting varying volumes of ethylene gas $(50-1000 \mu \mathrm{l})$ into an airtight cuvette. After incubation for $1 \mathrm{~min}$, the change in fluorescence was quantified. For experiments where RuQ was dissolved in pure organic solvent (100\% THF), reactivity was shown to be comparable to contemporary examples of ruthenium-based ethylene probes ${ }^{18,19}$. The limit of detection (LOD) was calculated to be $34.4 \mu \mathrm{l}$ of injected ethylene, which corresponds to about $27 \mathrm{ppm}$ in air. It should be noted that since physiological levels of ethylene produced during ripening are typically $<1 \mathrm{ppm}$, incubation periods for these probes would thus need to exceed times generally analogous with "real-time" detection (within $1 \mathrm{~min}$ ). In the case where experiments were performed for RuQ in an aqueous environment, reactivity was instead observed to become greatly diminished due to two main factors. First, it is an expected 
a
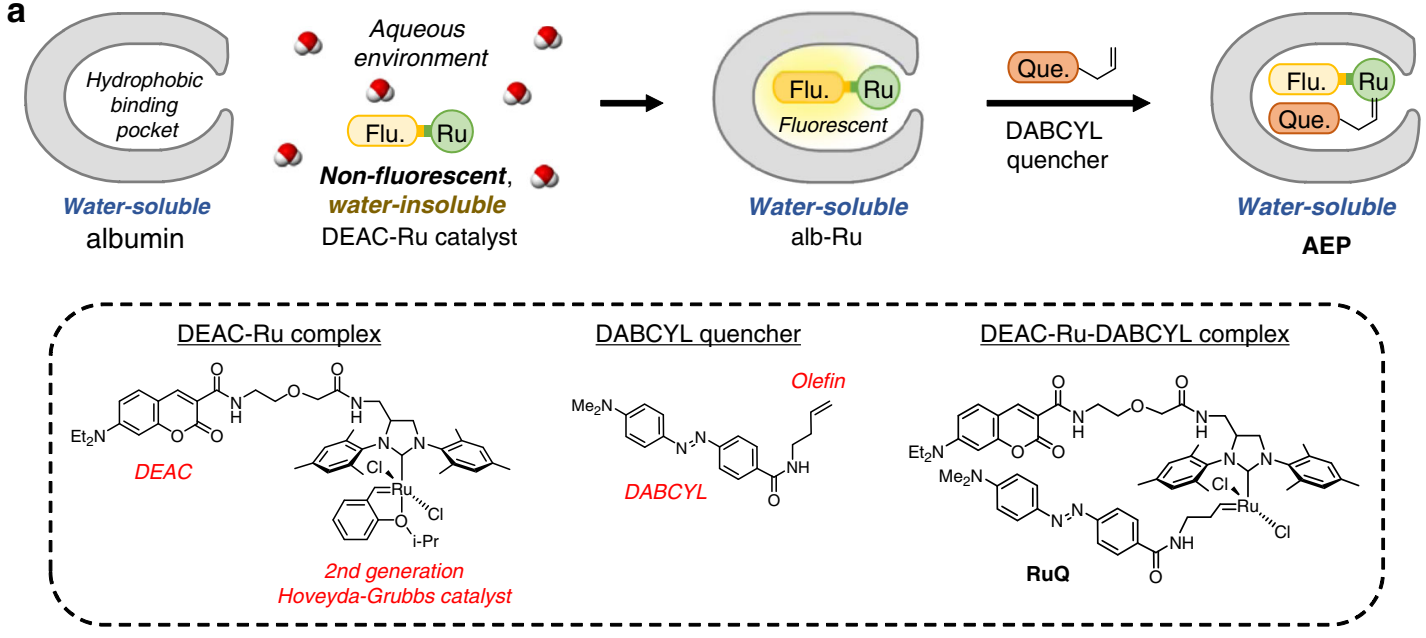

b

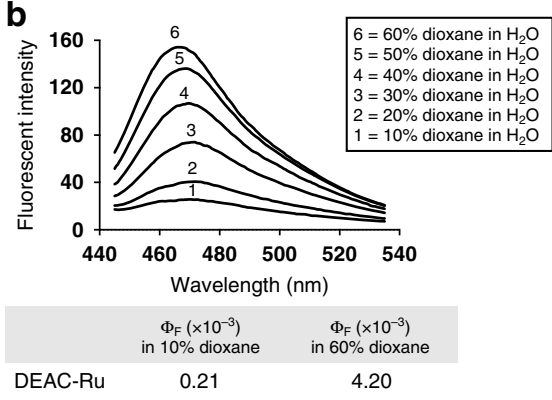

e

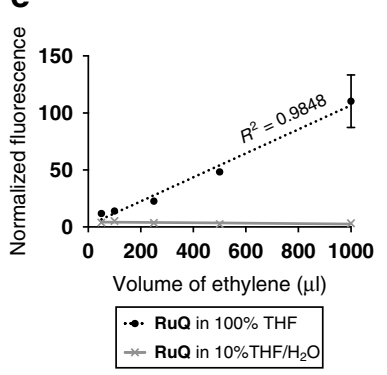

C

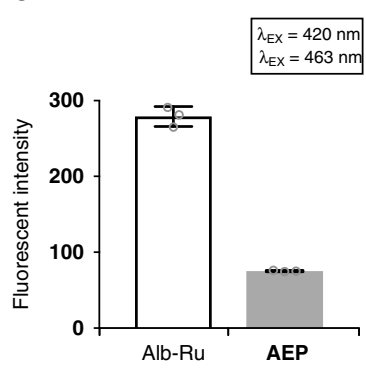

d

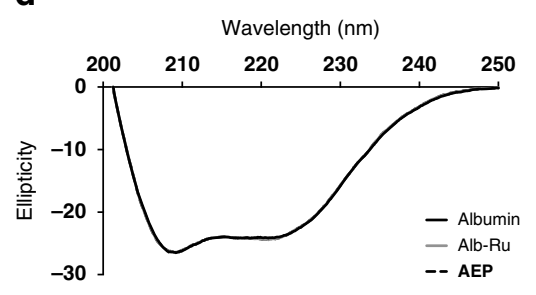

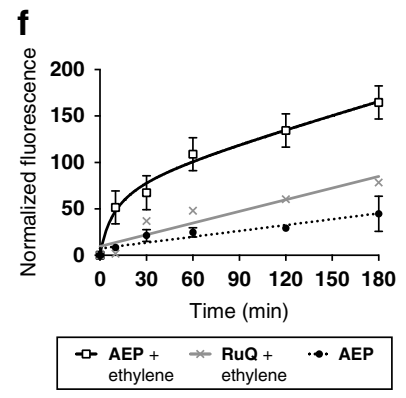

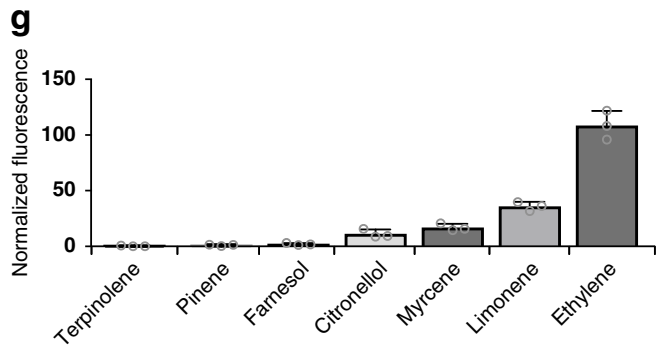

Fig. 2 Preparation and characterization of the AEP probe. a Depiction of the general steps taken to convert albumin into the ethylene detecting AEP probe. Chemical structures of the DEAC-Ru complex, DABCYL quencher, and RuQ are shown for reference. $\mathbf{b}$ Emission spectra and calculated quantum yields of DEAC-Ru under varying concentrations of dioxane in water. c Measured fluorescence of the alb-Ru complex compared to the non-fluorescent AEP probe. d Circular dichroism spectra comparison of the various protein complexes used in this study to investigate changes in structural folding. e Dose-dependent response curves for RuQ exposed to different volumes of ethylene gas under solvent conditions of either $100 \% \mathrm{THF}$ or $10 \% \mathrm{THF} / \mathrm{H}_{2} \mathrm{O}$. f Time-based monitoring of AEP $(100 \mu \mathrm{M})$ exposed to a theoretical concentration of $6 \mathrm{mM}$ ethylene dissolved in $\mathrm{H}_{2} \mathrm{O}$. $\mathbf{g}$ Selectivity study of AEP (100 $\mu \mathrm{M}$ ) against various types of terpenes $(6 \mathrm{mM})$. In general, fluorescence studies were performed at $\lambda_{\mathrm{EX}}=420 \mathrm{~nm} / \lambda_{\mathrm{EM}}=463 \mathrm{~nm}$. Error is represented as s.d. of three independent experiments. Source data are provided as a Source Data file.

occurrence that olefin metathesis using the Hoveyda-Grubbs catalyst proceeds with lower reactivities under aqueous conditions. And second, a limitation of the experimental setup does not properly account for the poor solubility of ethylene in water. To highlight this, NMR studies were done using similar air-to-liquid volume ratios (Supplementary Fig. 1). With $1 \mathrm{ml}$ of ethylene injected into pure solvent (100\% THF-d8), the dissolved concentration was calculated to be roughly $1 \mathrm{mM}$. However, under aqueous conditions $\left(25 \%\right.$ or $10 \%$ THF- $\left.d 8 / \mathrm{D}_{2} \mathrm{O}\right)$, no peaks corresponding to ethylene could be detected.

In order to design an experimental setup that can ensure an adequate level of ethylene is present under aqueous conditions to test AEP reactivity, the following assumptions were made. According to Henry's gas law, the amount of dissolved gas in a liquid is proportional to its partial pressure above the liquid. As such, one setup could see a closed system (with the cuvette) containing water that is purged and fitted with a balloon-containing ethylene gas. In this manner, it can be assumed that the only gas present is ethylene. Since the pressure of a balloon does not significantly deviate from 1 atm, it can be assumed that the total pressure of ethylene gas present is equivalent to its partial pressure. Using a Henry's law constant of $5.98 \times 10^{-3} \mathrm{M} \mathrm{atm}^{-1}$, the calculated concentration of dissolved ethylene in water would thus be approximately $6 \mathrm{mM}$, or 167 ppm. To initiate time-dependent reactivity studies, AEP was then injected into the ethylene-filled cuvette and monitored over time. Shown in Fig. 2f, a significant time-dependent increase in fluorescence was thus observed in the AEP/ethylene mixture compared to controls (RuQ/ethylene and AEP only).

Not only does ethylene has one of the highest reactivities for olefin metathesis ${ }^{43}$, but its smaller molecular size should allow it to 
a

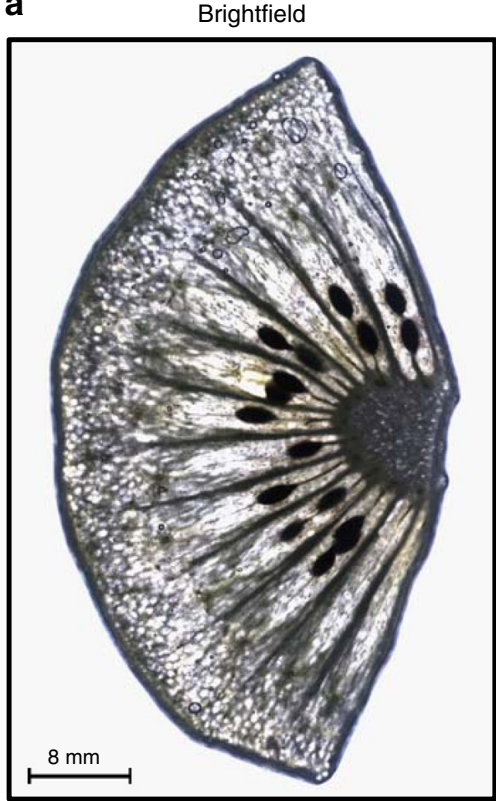

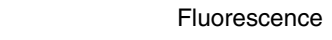
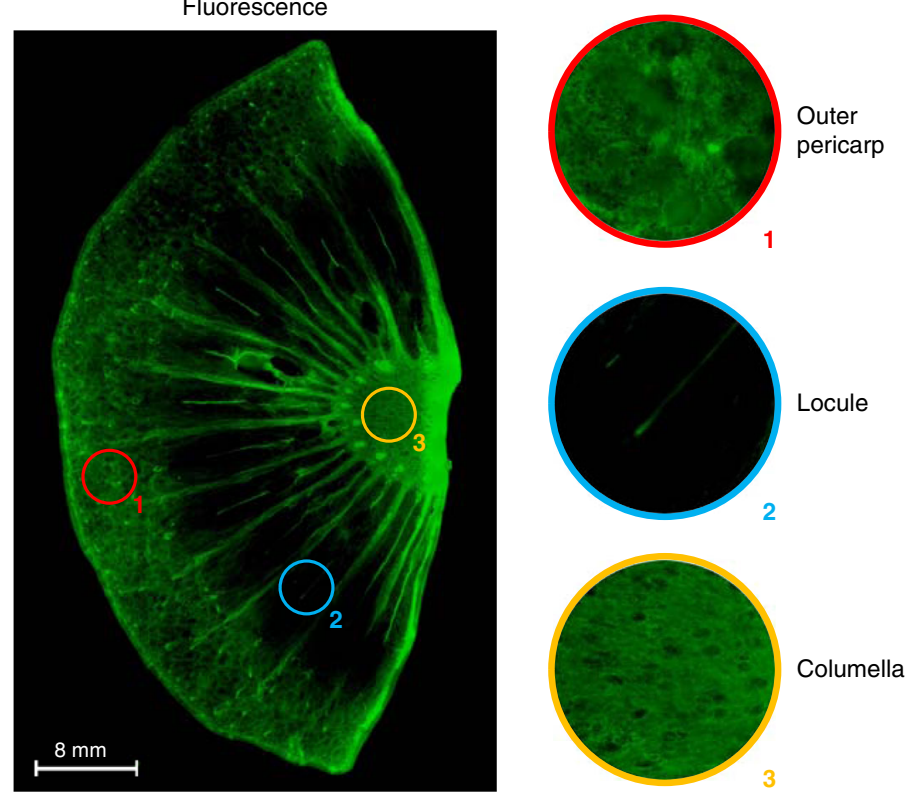

b
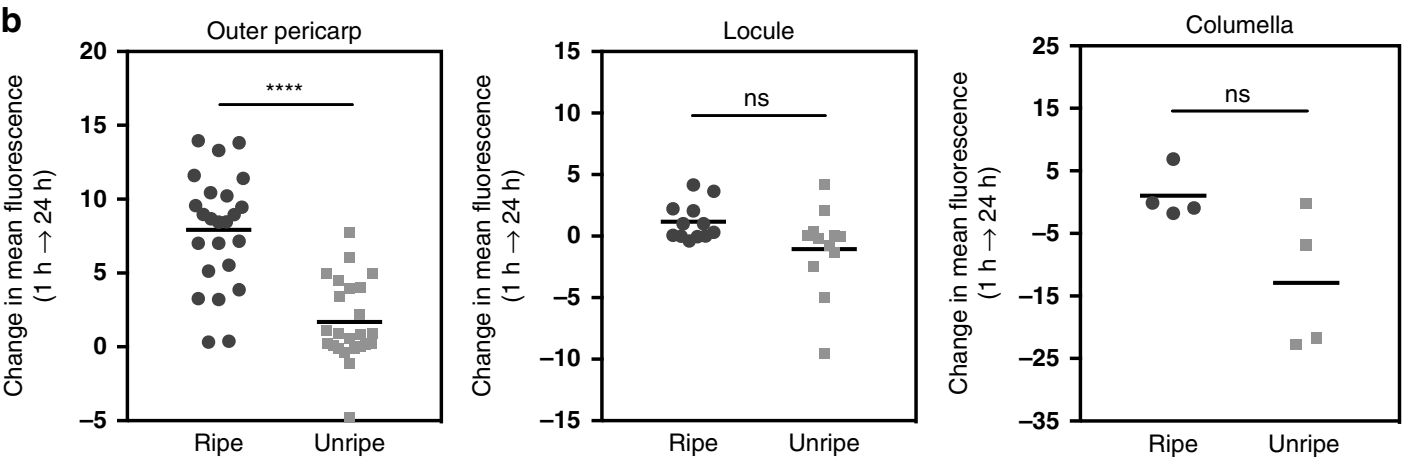

Fig. 3 Spatial-based detection of ethylene in ripening and unripe kiwifruit. a Fluorescent imaging of a ripening kiwi fruit, which was sectioned to display the outer pericarp, locules, and part of the columella. Multiple images were obtained at $\times 4$ magnification and were joined together via image stitching. b The measured change in fluorescence of the pericarp, locule, and the columella of kiwifruit (unripe or ripe) was taken between the 1 and $24 \mathrm{~h}$ timepoints. As shown, a significant increase in AEP-detected ethylene can be observed in the pericarp during the process of ripening. For each condition (unripe or ripe), two whole fruits were used to provide four different sections for investigation. Replicates values for the outer pericarp $(n=24)$, locule $(n=12)$, and the columella $(n=4)$ were obtained. Statistical analysis was performed using a paired sample $t$-test. ${ }^{\star} P<0.03,{ }^{\star \star} P<0.002,{ }^{\star \star \star} P<0.0002,{ }^{\star \star \star \star} P<0.0001$, $\mathrm{ns}=$ not significant. Source data are provided as a Source Data file.

enter the albumin-binding pocket with better ease compared to other plant metabolites, such as unsaturated molecules known as terpenes. To determine whether there is a chance for significant cross-reactivity with AEP, various terpenes (e.g. pinene, limonene, terpinolene, myrcene, citronellol, and farnesol) were tested utilizing similar conditions to the previous ethylene experiments (final terpene concentration of $6 \mathrm{mM}$ ). Shown in Fig. $2 \mathrm{~g}$, the overall reactivities of the tested terpenes were found to be significantly lower than with ethylene gas. However, it should be noted that in cases containing terminal unsaturated alkenes (such as myrcene and limonene), moderate activity was observed. As such, additional controls may be required when testing samples like cannabis (myrcene-abundant) and citrus fruits (limonene-abundant).

Spatial detection of ethylene in fruits. Climacteric fruits typically undergo autocatalytic ethylene (system 2) production during endogenous processes, such as ripening, and also in response to exogenous stimuli, such as wounding stress and pathogen infection $^{44,45}$. In this study, the spatial capabilities of the AEP probe was first investigated in response to endogenous ethylene. During ripening, studies have observed that kiwifruit exhibit an increase in ethylene production primarily through upregulation of ACS isogenes in the outer pericarp ${ }^{46}$. As shown in Fig. 3a and Supplementary Figs. 2-9, imaging experiments in kiwifruits using AEP focused on observing changes in ethylene expression of various organelles (outer pericarp, locule, and columella) at different stages of development (unripe vs. ripening). Summarized in Fig. 3b, a significant increase in fluorescence was detected in the outer pericarp from the transition of unripe to ripe fruit. On the other hand, fluorescent changes in the locule and columella of the kiwifruits did not occur at significant levels. As a note, it was observed that the tissues between individual locules generally exhibited an increase in fluorescence. Since literature has shown that kiwifruit seeds or surrounding tissues may play a role in ethylene biosynthesis (parthenocarpic kiwifruit are shown to have decreased levels of ethylene production due to suppressed expression of $\mathrm{ACS}^{47}$ ), a more thorough analysis may be of interest for future studies. Overall, with these results, the use of AEP presents itself as a promising tool to monitor the spatial expression of ethylene for fruits during ripening and development.

The ethylene biosynthetic pathway in plants (Fig. 4a) is mainly composed of three enzymes that convert methionine into 
a<smiles>CSCCC([NH3+])[O-]</smiles>

Methionine

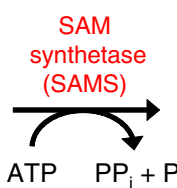

ATP $\quad P_{i}+P$

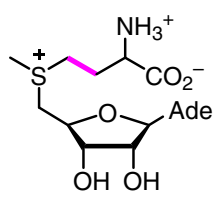

SAM

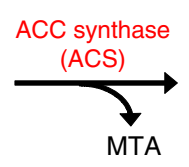

MTA

Ethylene biosynthetic pathway

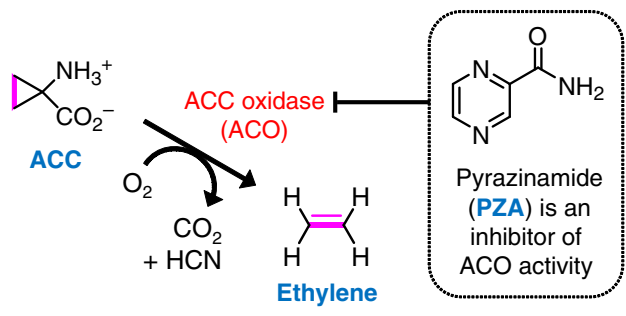

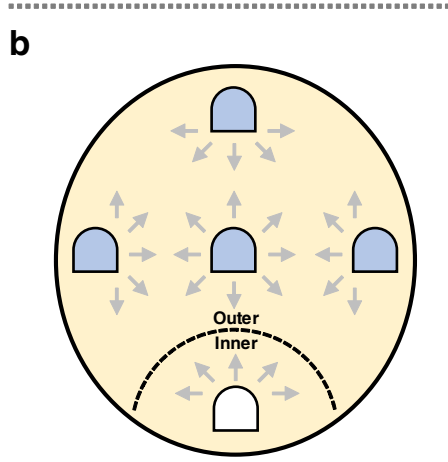
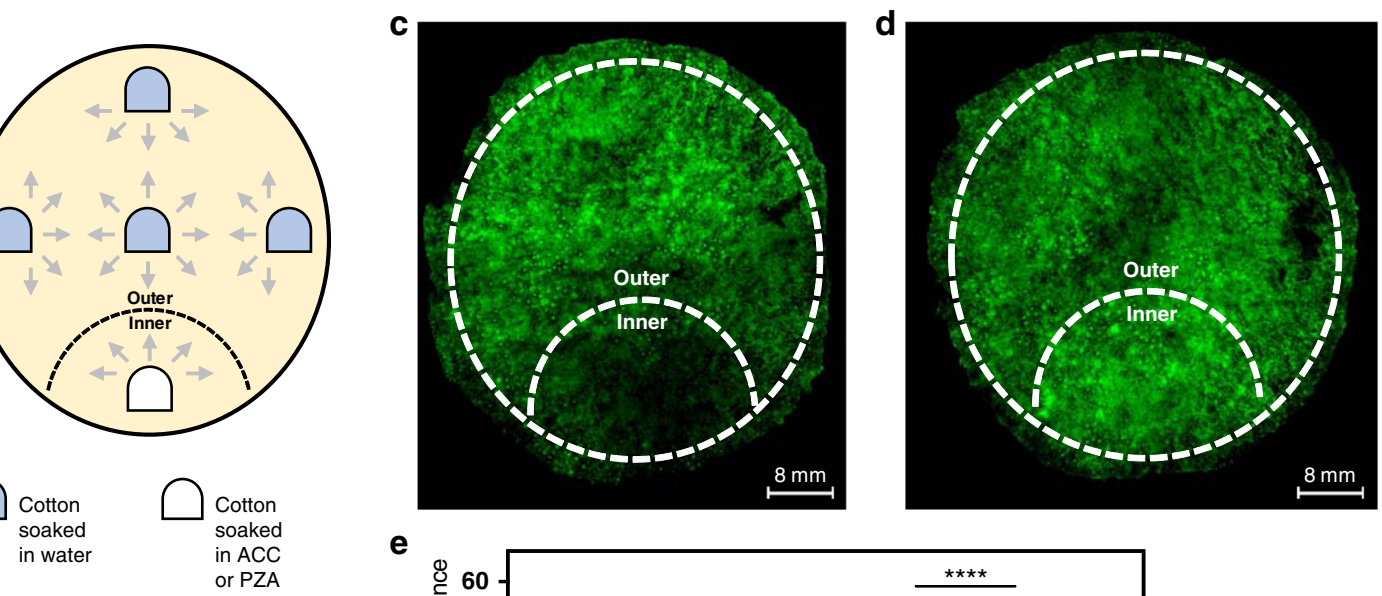

e

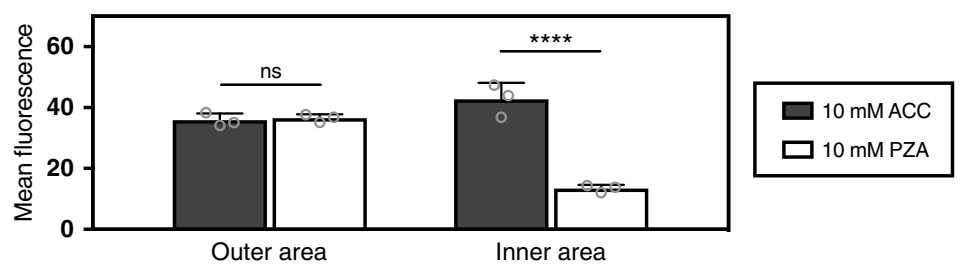

Fig. 4 Spatial-based detection of ethylene in sections of Asian pears induced with either an ethylene-stimulating or ethylene-inhibiting additive. a Scheme of the ethylene biosynthetic pathway in plants. Addition of ACC, the metabolite directly preceding ethylene biosynthesis, will stimulate ethylene production. Addition of PZA, a known inhibitor of ACC oxidase, will decrease ethylene production. $\mathbf{b}$ Cotton balls soaked in specific solutions (water, ACC, or PZA) are gently applied to sections of Asian pears. To minimize diffusion, five cotton balls are applied with equal spacing to the surface of pear sections. Four of them contain water, while one of them has been soaked in either $10 \mathrm{mM} \mathrm{ACC}$ or $10 \mathrm{mM}$ PZA. c Fluorescent imaging of an Asian pear section where the inner area has been exposed to $10 \mathrm{mM} \mathrm{PZA}$. d Fluorescent imaging of an Asian pear section where the inner area has been exposed to $10 \mathrm{mM}$ ACC. e Summary of measured fluorescence between the outer and inner areas of Asian pear sections that have been supplemented with either ACC or PZA Multiple images were obtained at $\times 4$ magnification and were joined together via image stitching. Statistical analysis was performed using a one-way ANOVA with Tukey's multiple comparisons test. ${ }^{\star} P<0.03,{ }^{\star \star} P<0.002,{ }^{\star \star \star} P<0.0002,{ }^{\star \star \star \star} P<0.0001$, ns $=$ not significant. Error is represented as s.d. of three independent experiments. Source data are provided as a Source Data file.

ethylene: SAM synthetase (SAMS), ACC synthase (ACS), and ACC oxidase $(\mathrm{ACO})^{48}$. Although ACS is often regarded as the rate-limiting step of ethylene biosynthesis ${ }^{49}$, recent research has indicated that ACO may also be rate limiting during postclimacteric ripening of tomatoes (and likely other climacteric fruit $)^{50,51}$. Of relative importance to this study, pyrazinamide (PZA) was recently found to be an inhibitor of ethylene biosynthesis through inhibition of $\mathrm{ACO}^{52}$. With consideration of this pathway, this study generally relied on using ACC as a stimulant of ethylene production, and PZA as a repressor of ethylene production.

In another investigation, the ability of AEP to respond to changes in ethylene biosynthesis due to external stimuli was carried out. Cotton balls soaked in either a solution of ACC or PZA were topically applied to sections of fruit flesh (hypanthium) from Asian pears. In order to ensure uniform hydration of the sample, waterinfused cotton balls were placed in an equally spaced arrangement depicted in Fig. 4b. Following a $5 \mathrm{~h}$ incubation period, cotton balls were removed and the samples were then placed on plates preloaded with AEP. Fluorescence microscopy-imaging results obtained after $18 \mathrm{~h}$ are shown in Fig. 4c, d and Supplementary Figs. 10 and 11. In this study, the inner area is defined as the tissue roughly within a $15 \mathrm{~mm}$ radius from the origin of stimulant (ACC or PZA) application. On the other hand, the outer area is the tissue area exposed to water (as a control). As summarized in Fig. 4e, results clearly show differences in inner area fluorescence compared to outer area fluorescence. For example, exposure to ACC was shown to moderately increase inner area fluorescence by about $20 \%$. Alternatively, exposure to PZA gave the opposite effect by greatly decreasing inner area fluorescence by up to $60 \%$. Although promising, it should be noted that there are major experimental challenges associated with studying spatial changes in ethylene biosynthesis due to external stimuli; the main culprit being the issue of diffusion. Without a strong external inducer, diffusion of the stimulant greatly decreases spatial resolution. This is especially problematic for samples with smaller surface areas, as similar experiments could not be reproduced with smaller plant leaves.

Temporal detection of ethylene in fruits. To investigate the temporal detection capabilities of the AEP probe across a variety of different fruits, the general protocol outlined in Fig. 5a was followed. To begin, small sections of fruit measuring roughly $10 \mathrm{~mm} \times 8 \mathrm{~mm}$ in size were prepared. These samples were then incubated with varying concentrations of ACC or PZA for $3 \mathrm{~h}$ at 
a
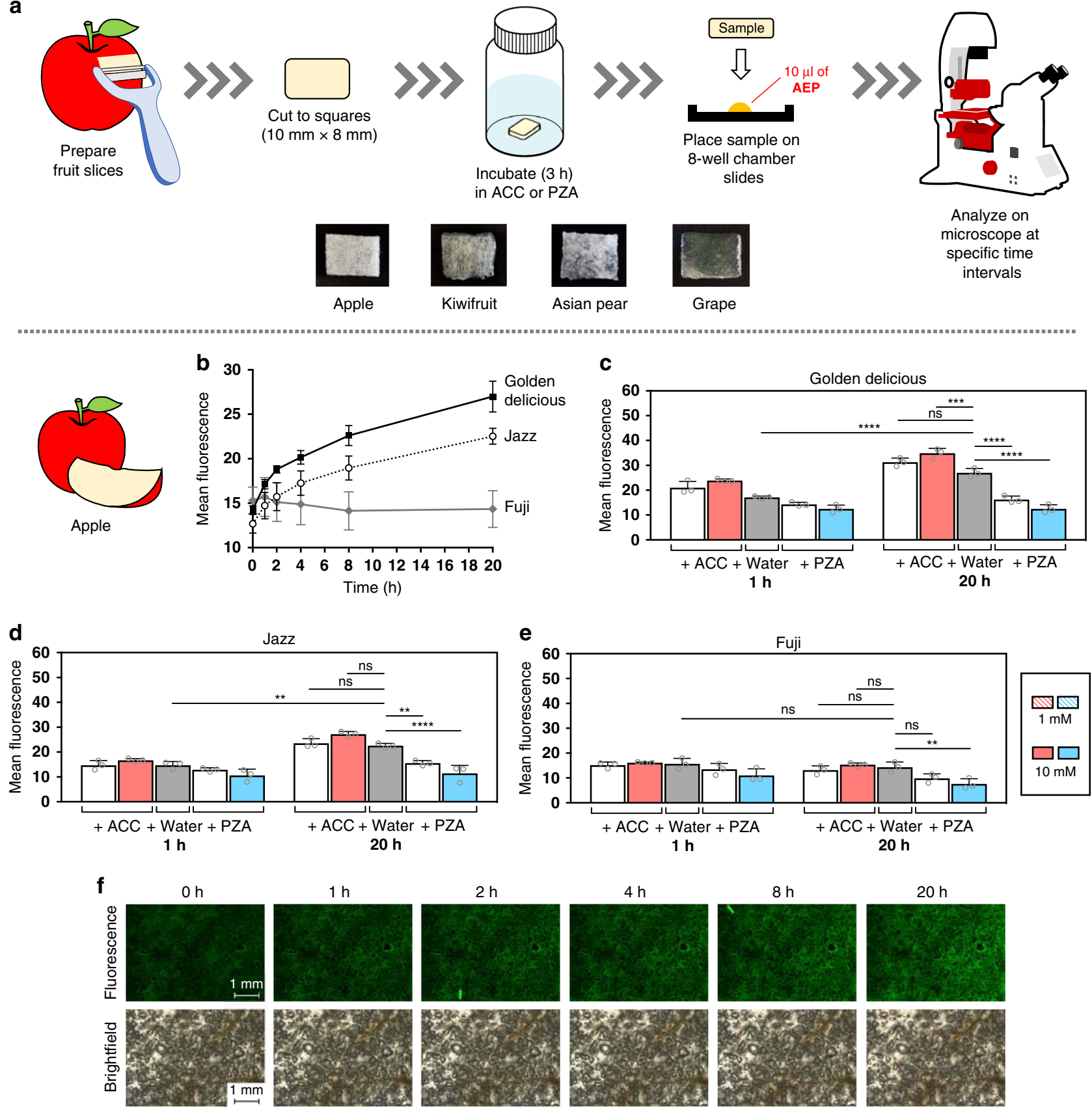

Fig. 5 Time-dependent detection of ethylene in apples. a In general, samples were prepared from slices of fruit flesh cut into $\sim 10 \mathrm{~mm} \times 8 \mathrm{~mm}$ segments. Following a $3 \mathrm{~h}$ incubation either in solutions of ACC, PZA, or water (as a control), samples were then removed and placed onto chamber slides preloaded with AEP probe ( $10 \mu \mathrm{l}$ of a $400 \mu \mathrm{M}$ solution). Using an inverted microscope, samples were monitored at specific time intervals. To examine the difference in ethylene production across different apple cultivars, fluorescence was monitored over time for Golden Delicious, Jazz, and Fuji apples b. A summary and statistical comparison between the 1 and $20 \mathrm{~h}$ timepoints for each apple cultivar are also shown c-e, as well as samples images of Golden Delicious apples (water-control) over various timepoints $\mathbf{f}$. Statistical analysis was performed using a one-way ANOVA with Tukey's multiple comparisons test. ${ }^{\star} P<0.03$, ${ }^{\star \star} P<0.002,{ }^{\star \star \star} P<0.0002,{ }^{\star \star \star \star} P<0.0001, \mathrm{~ns}=$ not significant. Error is represented as s.d. of three independent experiments. Source data are provided as a Source Data file.

room temperature, before being placed onto chamber slides preloaded with AEP. Fluorescence analysis was then carried out at specific time intervals.

In literature, internal ethylene levels have been shown to vary among different types of apple cultivars ${ }^{53-55}$. For instance, Fuji apples are known to produce lower levels of ethylene gas compared to other cultivars ${ }^{55}$. To test the capacity of AEP to detect these differences, time-based studies were carried out using apple cultivars that included Golden Delicious, Jazz, and Fuji apples (Fig. 5b-f and Supplementary Figs. 12-26). As summarized in Fig. 5b, data clearly shows that Fuji apples produce lower levels of ethylene compared to other cultivars over time. Furthermore, this trend was also observed during supplementation studies using ACC or PZA (Fig. 5c-e).

Additionally, other fruits were also examined in this study using the AEP probe, which included kiwifruit, Asian pears, and Muscat grapes (Fig. 6 and Supplementary Figs. 27-41). For climacteric fruits like kiwifruit and Asian pears ${ }^{56,57}$, both 


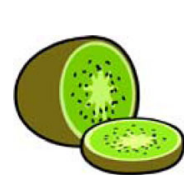

Kiwifruit

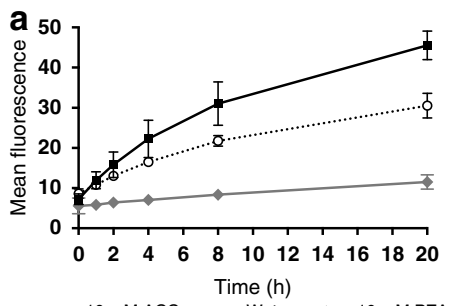

$\rightarrow 10 \mathrm{mM} \mathrm{ACC} \cdots . \cdots$ Water $\multimap 10 \mathrm{mM}$ PZA
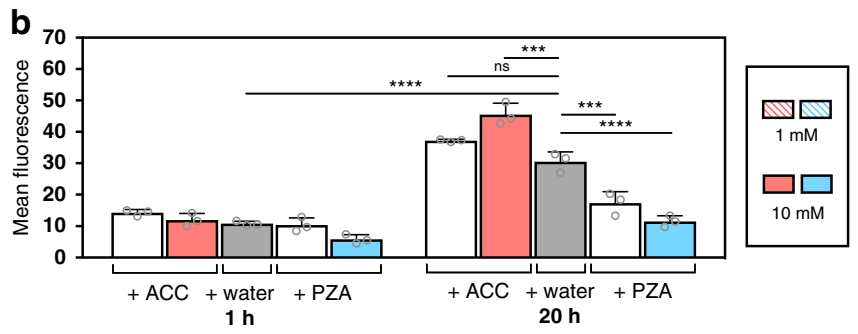

C

$\mathrm{Oh}$
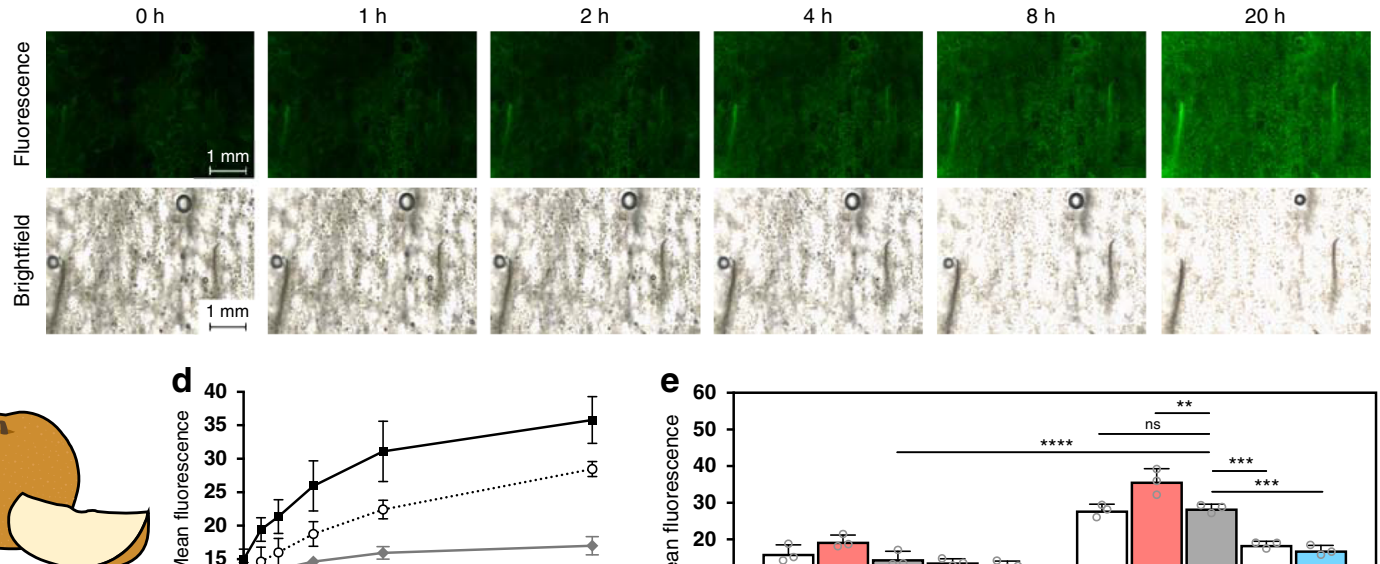

Asian pear
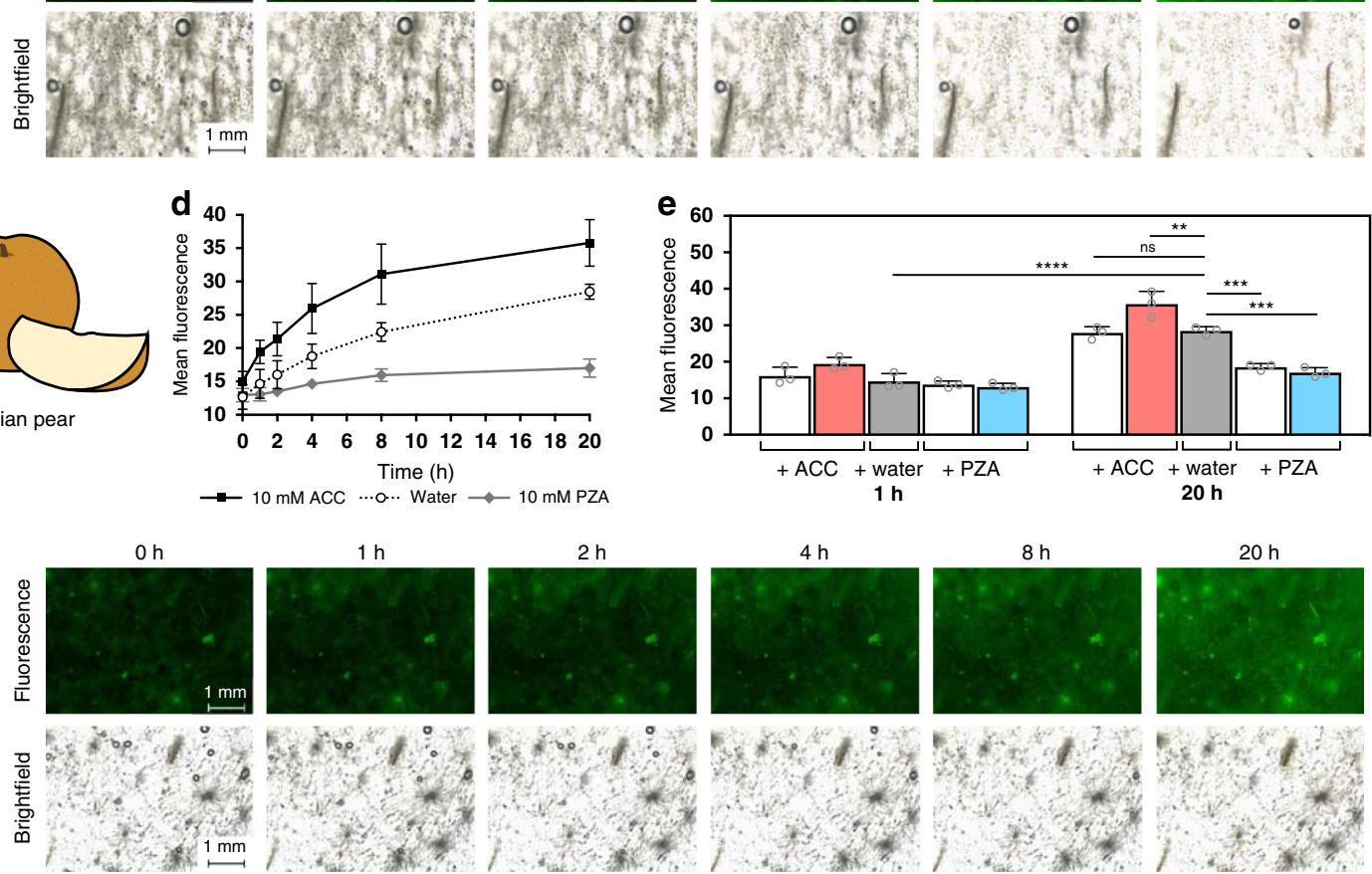

f
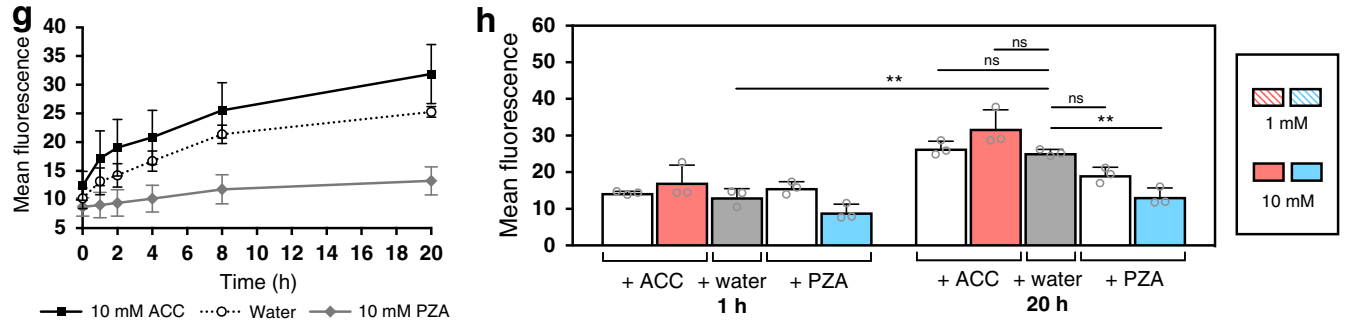

i
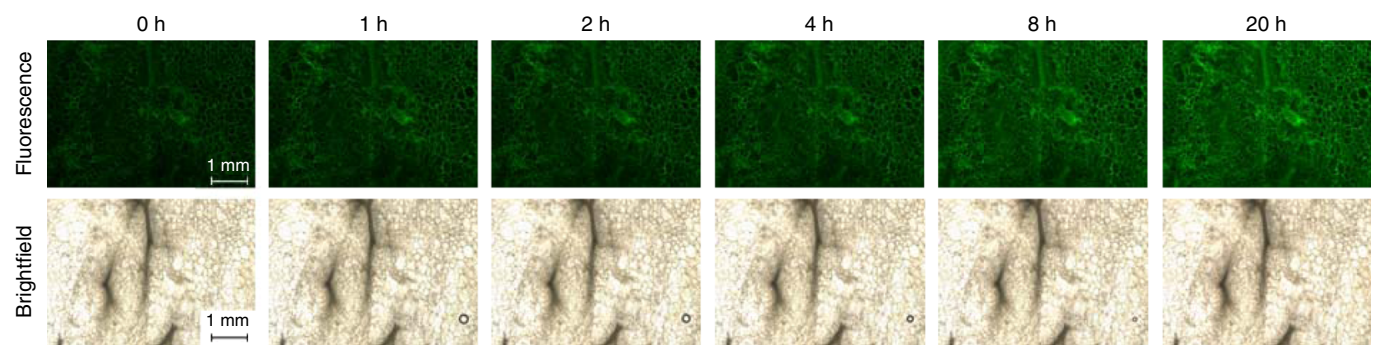

Fig. 6 Time-dependent detection of ethylene in other ethylene-producing fruits. Fluorescence was monitored over time under various conditions (ACC or PZA) for kiwifruit $\mathbf{a}$, Asian pear $\mathbf{d}$, and Muscat grapes $\mathbf{g}$. A summary and statistical comparison between the 1 and $20 \mathrm{~h}$ timepoints for each fruit are shown $\mathbf{b}, \mathbf{e}, \mathbf{h}$, as well as samples images (water-control) over various timepoints $\mathbf{c}$, $\mathbf{f}$, i. Statistical analysis was performed using a one-way ANOVA with Tukey's multiple comparisons test. ${ }^{\star} P<0.03$, ${ }^{\star \star} P<0.002,{ }^{\star \star \star} P<0.0002,{ }^{\star \star \star \star} P<0.0001$, ns $=$ not significant. Error is represented as s.d. of three independent experiments. Source data are provided as a Source Data file. 


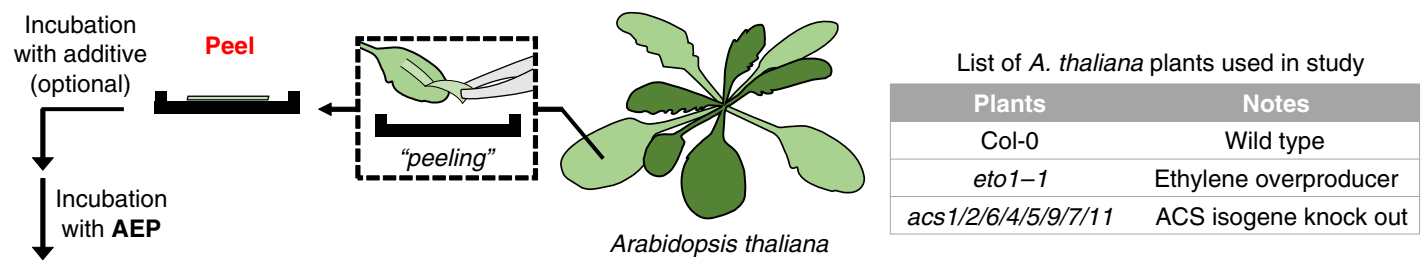

b

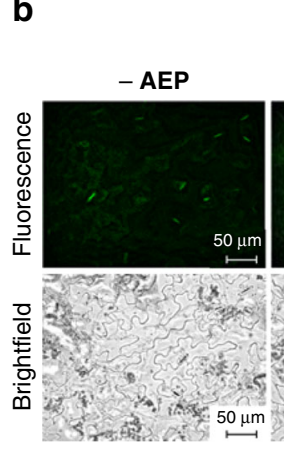

d

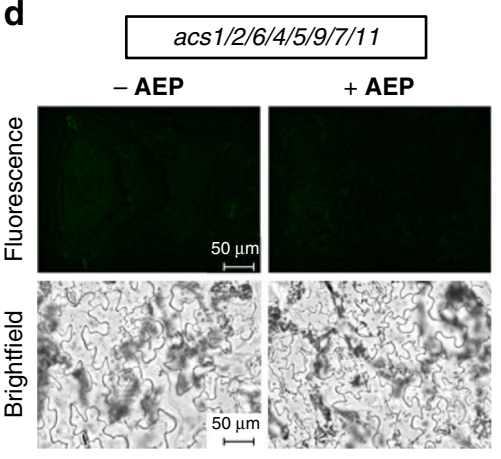

Col-0

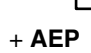

$-\mathrm{AEP} /+\mathrm{ACC}$
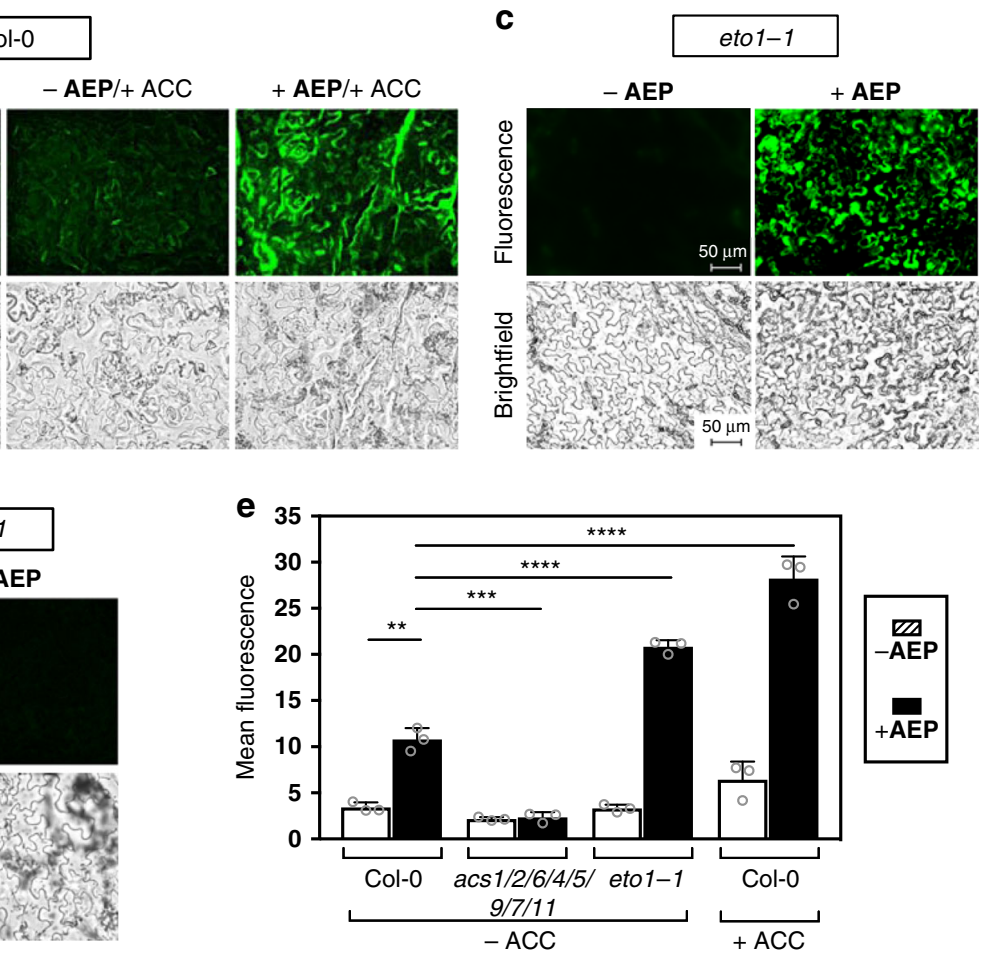

Fig. 7 Detection of ethylene produced in $\boldsymbol{A}$. thaliana plants. a Depiction of sample preparations to obtain epidermal peels from $A$. thaliana and the list of plants used in this experiment. Fluorescence and brightfield imaging ( $\times 40$ magnification) of epidermal peels applied with AEP (100 $\mu$ M) for wild-type Col-0 b, ethylene overproducer eto1-1 $\mathbf{c}$, and the ethylene biosynthesis defected acs $1 / 2 / 6 / 4 / 5 / 9 / 7 / 11$ d. Additionally, supplementation studies were also performed using the ethylene biosynthetic precursor ACC (1 mM), which was only applied to wild-type Col-0. e Summary of the measured fluorescence between the various $A$. thaliana plants applied with and without AEP, as well as the effects of ACC supplementation. Statistical analysis was performed using a one-way ANOVA with Tukey's multiple comparisons test. ${ }^{\star} P<0.03,{ }^{\star \star} P<0.002,{ }^{\star \star \star} P<0.0002,{ }^{\star \star \star \star} P<0.0001$, ns $=$ not significant. Error is represented as s.d. of three independent experiments. Source data are provided as a Source Data file.

displayed time-dependent ethylene production as expected. Interestingly, samples from Muscat grapes were also tested and produced ethylene in a time-dependent manner. Although generally classified as non-climacteric, studies have shown grapes have the capacity to produce ethylene gas; reaching its peak during grape maturation ${ }^{58}$. Furthermore, detectable levels of ACS and ACO gene expression can be found long after development and ripening ${ }^{59}$. As a step to further check AEP sensitivity, negative controls were carried out with ethylene non-producing vegetables like carrots and red bell peppers (Supplementary Figs. 42-52). As expected, these cases produced non-significant levels of ethylene over similar time periods.

Ethylene detection in plants. To investigate the efficacy of AEP detection in plants, the small flowering plant Arabidopsis thaliana (Brassicaceae family) was chosen as the model organism. Focusing specifically on imaging of clear epidermal peels, comparative studies were carried out using a wide range of small molecules and plant mutants (Fig. 7 and Supplementary Figs. 53 and 54).

For this study, the Col-0 ecotype of $A$. thaliana served as the model wild-type plant. Also used were various mutants; acs1/2/6/
4/5/9/7/11 and eto1-1. The mutant acs 1/2/6/4/5/9/7/11, which contains knock outs of eight functional ACS genes, was chosen because it exhibits dramatically reduced levels of ethylene that persist even during pathogen invasion ${ }^{60,61}$. This is due to the key role that ACS plays in the ethylene biosynthetic pathway (Fig. 4a). Another of the chosen mutants is eto1-1, which is characterized by ethylene overproduction ${ }^{62}$. In this case, the eto $1-1$ mutation results in the stabilization of type II ACS, thereby preventing proteasomal degradation of $\mathrm{ACS}^{63}$.

Shown in Fig. $7 \mathrm{~b}$ are the images obtained for Col-0 incubated with and without AEP at room temperature. As quantified in Fig. 7e, a significant increase in fluorescence was observed, thereby validating the use of AEP for ethylene detection in $A$. thaliana epidermal peels. As a positive control, ethylene production was contrasted with Col-0 exogenously stimulated by ACC incubation. As expected, AEP-based ethylene detection showed a significantly higher increase in detected fluorescence compared to the wild type Col-0.

To further evaluate the sensitivity of the AEP probe to detect endogenous differences in ethylene production, comparative studies were then carried out using mutants acting as either a positive ethylene control (eto1-1) or a negative ethylene control 
(acs1/2/6/4/5/9/7/11). Shown in Fig. 7c, imaging for the ethylene overproducer, eto1-1, showed a significant increase in fluorescence compared to the wild type Col- 0 , as expected. In contrast, with the acs $1 / 2 / 6 / 4 / 5 / 9 / 7 / 11$ mutant that naturally lacks the ability to produce ethylene, there was no discernible fluorescent change with AEP addition (Fig. 7d). When compared with the wild-type Col-0, the acs knockout plant thus exhibited significantly lower levels of fluorescence.

In the next part of this study, AEP probe sensitivity was tested in response to environmental stress factors, namely the immunogenic response to pathogen invasion in A. thaliana. Focus will be given to changes in ethylene production caused by two separate plant immunity pathways: PAMP-triggered immunity (PTI) and effector-triggered immunity (ETI).
PTI-based ethylene detection. Pathogenic bacteria attempting to invade plant cells initially must contend with the first tier of plant's defense mechanism-PTI. Depicted in Fig. 8a, this system is triggered by the recognition of pathogen-derived molecules known as pathogen-associated molecular pattern (PAMP) through pattern recognition receptors (PRRs) on the plasma membrane. Once triggered, a signaling cascade then commences to upregulate genes associated with the defense response. Among the upregulated genes, studies have shown that ethylene biosynthesis plays an integral role in $\mathrm{PTI}^{64}$, possibly due to its regulatory role for innate immune receptors ${ }^{65}$.

Two well-studied PAMPs used in this study are the flg22 and elf18 bacterial PAMPs. Flagellin is a globular protein found in the filament that composes the bacterial flagellum. On the conserved
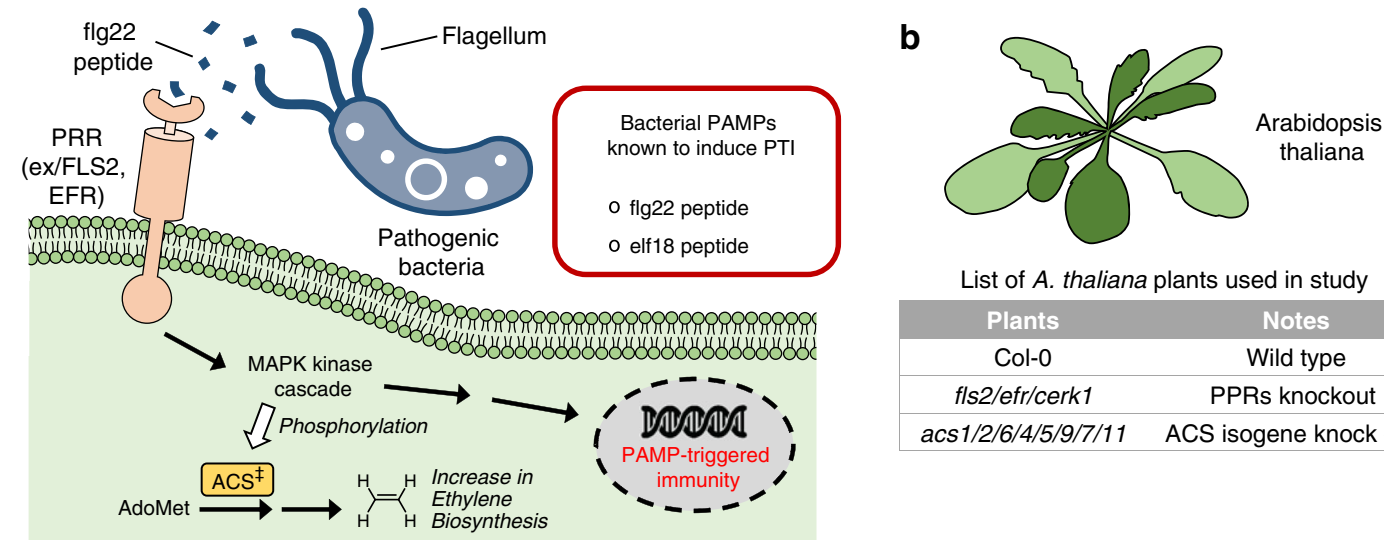

List of $A$. thaliana plants used in study

\begin{tabular}{|cc|}
\hline Plants & Notes \\
\hline Col-0 & Wild type \\
\hline fls2/efr/cerk1 & PPRs knockout \\
\hline acs $1 / 2 / 6 / 4 / 5 / 9 / 7 / 11$ & ACS isogene knock out ${ }^{\ddagger}$ \\
\hline
\end{tabular}

C

C

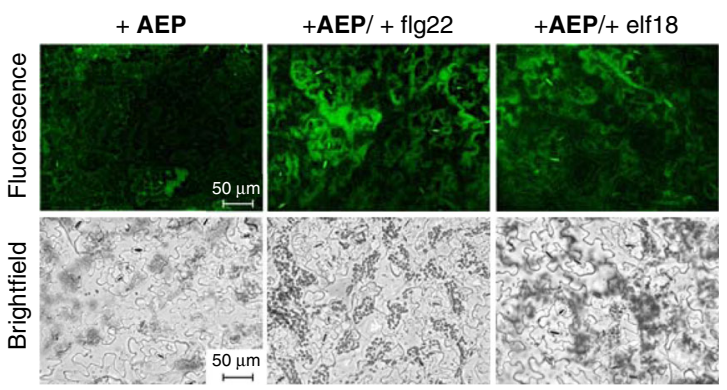

e

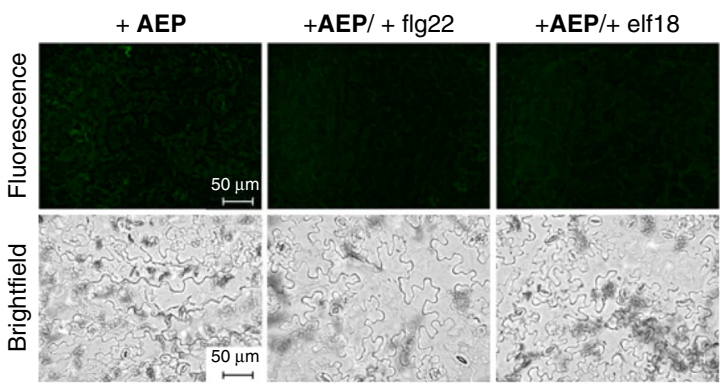

d
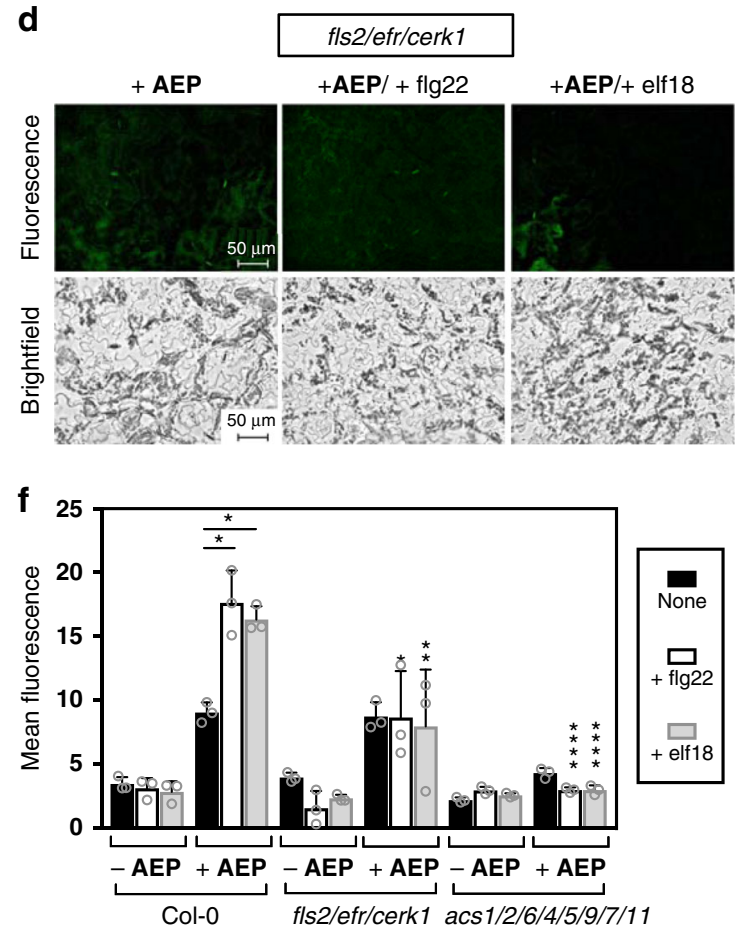

Fig. 8 Detection of ethylene produced as a result of PAMP-triggered immunity in A. thaliana plants. a Depiction of the general pathway that leads to PTI response. $\mathbf{b}$ List of $A$. thaliana plants used in this experiment. Fluorescence and brightfield imaging ( $\times 40$ magnification) of epidermal peels applied with $\mathbf{A E P}$ $(100 \mu \mathrm{M})$ for wild-type Col-0 c, PRR-deficient fls2/efr/cerk1 d, and the ethylene biosynthesis defected acs1/2/6/4/5/9/7/11 e. Additionally,

supplementation studies were performed using the PAMPs, flg22, and elf18 $(4.8 \mu \mathrm{M})$, which were applied to all plants. $\mathbf{f}$ Summary of the measured fluorescence between the various conditions studied. Statistical analysis was performed using a one-way ANOVA with Tukey's multiple comparisons test. If not indicated, comparisons were made between the mutants and wild-type under the same treatment conditions. ${ }^{\star} P<0.03,{ }^{\star \star} P<0.002,{ }^{\star \star \star} P<0.0002$, ${ }^{\star * \star \star} P<0.0001, \mathrm{~ns}=$ not significant. Error is represented as s.d. of three independent experiments. Source data are provided as a Source Data file. 
$\mathrm{N}$-terminus of flagellin, a 22-amino acid sequence known as flg22 has been found to activate plant defense mechanisms ${ }^{66,67}$. It does this by first binding to the PRR known as FLS2, which then leads to the activation of MAPK-signaling cascades and the production of reactive oxygen species (ROS) to confer pathogen resistance ${ }^{68}$. For the bacterial protein EF-Tu (elongation factor thermo unstable), an 18-amino acid sequence, termed elf18, is known to be important for post-invasive immunity and capable of eliciting $\mathrm{PTI}^{69}$. In this case, perception instead acts through the PRR known as EFR (EF-TU receptor), followed by downstream MAPK signaling and ROS production ${ }^{70}$.

In this study, epidermal peels from $A$. thaliana Col-0 were first incubated for $6 \mathrm{~h}$ with either PAMP (flg22 or elf18) or a blank control. Incubation with AEP was then carried out, followed by imaging and quantification (Fig. 8c-e and Supplementary Figs. 55-60). As shown in Fig. 8c, significant increases in fluorescence could be detected with tissues incubated with either PAMP compared to the blank control. This data correlates well with literature reports that have shown consistently high levels of ethylene gas generated after flg22 or elf18 exposure ${ }^{61}$.

As a further investigation, control studies were also carried out using the mutant plants $f l s 2 / e f r / c e r k 1$ and $a c s 1 / 2 / 6 / 4 / 5 / 9 / 7 / 11$. For the $A$. thaliana mutant known as $f l s 2 /$ efr/cerk 1 , the two PAMPsensing PRR genes FLS2 and EFR are knocked out, leaving these plants unable to sense and respond to the presence of extracellular PAMP. With the images shown in Fig. 8d, fluorescence levels in the mutants incubated with either flg22 or elf18 falls to levels on par with the blank controls, thereby representing a significant decrease compared to the corresponding wild type Col- 0 . With the acs $1 / 2 / 6 / 4 / 5 / 9 / 7 / 11$ mutant, no discernible difference in fluorescence with epidermal peels incubated with or without PAMPs was found (Fig. 8e). Consistent with the fact that this mutant is unable to biosynthesize ethylene $^{60,61}$, observed fluorescence was again significantly reduced compared to the corresponding wild type Col- 0 .

ETI-based ethylene detection. In case the first defense system (PTI) is defeated, innate resistance mechanisms in plants initiate a second defense system known as ETI. As depicted in Fig. 9a, the basis behind this immune response relies on the fact that bacterial pathogens are known to infect eukaryotic cells using an appendage to secrete toxins or effectors into the host cells by type III secretion system (or injectisome). These secreted effectors have the ability to exert a number of effects that promote pathogen virulence. Examples of well-studied effectors are AvrRpm1 and AvrRpt2, which can be produced by Pseudomonas syringae pv. tomato (Pst) to interfere with the PTI-regulating membrane protein RPM1-interacting protein 4 (RIN4) $^{71}$. To combat this, resistant plants have resistance $(\mathrm{R})$ proteins that recognize directly or indirectly some of these effectors (known as avirulent effectors), leading to a signaling cascade that upregulates genes associated with the defense response. For AvrRpm1, once associated with RIN4, phosphorylation of RIN4 is induced, followed by activation of the $\mathrm{R}$ protein known as $\mathrm{RPM} 1^{72}$. In the case of AvrRpt2, degradation of RIN4 occurs instead. Since the R-protein RPS2 also associates with RIN4, its degradation essentially frees RPS2 to interact and be activated by AvrRpt2 ${ }^{73,74}$.

In this study, ETI-induced ethylene production was monitored in A. thaliana via exposure to two different bacterial strains known to produce either the AvrRpm1 or AvrRpt2 avirulence proteins. To carry out this study, inoculation of epidermal peels with Pst AvrRpm1, Pst AvrRpt2, or a $10 \mathrm{mM} \mathrm{MgCl}_{2}$ solution was first done $12 \mathrm{~h}$ before detection. Incubation with AEP was then carried out, followed by imaging and quantification (Fig. 9c-e and Supplementary Figs. 61-66). As shown in Fig. 9c, the Col-0 epidermal peels that were exposed to Pseudomonas producing either AvrRpm1 or AvrRpt2 showed significantly increased levels of fluorescence compared to the $\mathrm{MgCl}_{2}$ control.

Again, as a comparative study, A. thaliana mutants were also tested as controls to highlight the robustness of the AEP probe. One of the mutants used was rpm1rps2, which has both the $\mathrm{R}$ proteins, RPM1 and RPS2, knocked out. In this regard, the rpm1rps 2 mutant is unable to properly activate ETI even after infection with Pseudomonas bacteria carrying AvrRpm1 or AvrRpt2. Shown in Fig. 9d, imaging results clearly show that fluorescence levels in the mutant plant exposed to either AvrRpm1 or AvrRpt2 did not have elevated levels of ethylene production. Instead, fluorescence levels remain on par with the $\mathrm{MgCl}_{2}$ controls. In comparison to the corresponding wild type Col-0, this signifies a dramatic reduction in detected ethylene. Finally, in the case with the acs $1 / 2 / 6 / 4 / 5 / 9 / 7 / 11$ mutant, no discernible differences in fluorescence was found with exposure to AvrRpm1 or AvrRpt2 (Fig. 9e), which agrees well with literature ${ }^{61}$.

Limitations. Through testing numerous different samples in this study, some limitations of the AEP probe were gradually noted. First, one key issue is absorption. Given that the exterior scaffold of AEP is human serum albumin ( $66 \mathrm{kDa}$ protein), the probe is unlikely to passively penetrate across the cell membrane and into plant cells. It is assumed that the AEP probe exists extracellularly, rather than intracellularly. Due to this, the texture of a sample becomes a limiting factor. When used with absorbent samples, such as the flesh of fruits, the AEP probe was observed to sufficiently remain on sample surfaces. However, when testing with samples of waxy nature (i.e. plant cuticles), it was noted that the AEP probe tends to get pushed off over time.

Another issue is related to the aspect of "real-time" detection. Due to its poor water solubility, ethylene rapidly transitions from solution into the atmosphere. As such, any (water-based) bioimaging reagent used to directly measure this phytohormone in "real-time" must react under a very short period of time. At its current state, however, AEP probe reactivity is not fast enough to measure "real-time". Conceivable approaches to improve upon this area could see changes made to (1) increase ruthenium reactivity by altering the metal-ligand complex, (2) change the protein scaffold for one with a shallow-binding pocket to allow easier access, or (3) adopt the use of a stronger FRET donor-acceptor pair.

\section{Discussion}

Overall, the work conducted in this study should have a significant impact for the advancement of two separate research fields, namely in the areas of enzyme biosensor development and also research focused on ethylene detection in plants.

Given the biocompatible nature of enzyme biosensors, their usage for biomedical applications not only has implications from a scientific perspective, but also from a commercial standpoint. Currently, numerous examples of enzyme biosensors are applied commercially, such as arylacylamidase, $\beta$-hydroxybutyrate dehydrogenase, salicylate hydroxylase, and uricase, which are used for the serum determination of metabolites like acetaminophen, ketone bodies, salicylate, and uric acid, respectively. However, since enzyme biosensors are mainly derived from naturally occurring enzymes, recent progress in this field has largely appeared to stall. In parallel, a rapid rise of interest is now being directed towards the use of biosensors composed of abiotic metal complexes, where the bioorthogonality of certain transition metals can help to target metabolites typically found in difficultto-access chemical spaces. 
a

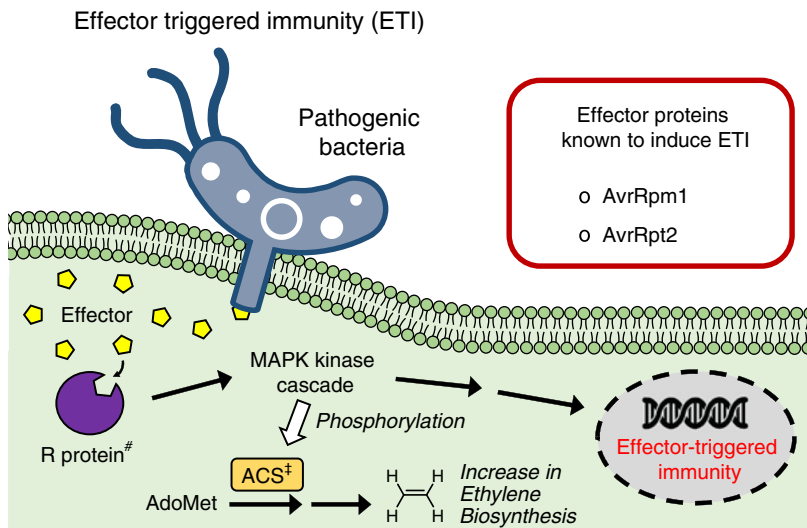

b

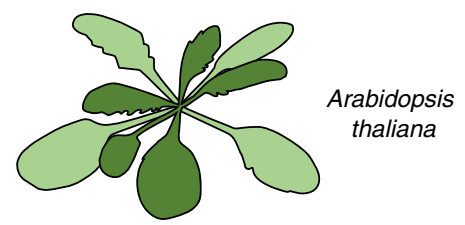

List of $A$. thaliana plants used in study

\begin{tabular}{|cc|}
\hline Plants & \multicolumn{1}{|c|}{ Notes } \\
\hline Col-0 & Wild type \\
\hline rpm1rps2 & R protein knock out ${ }^{\#}$ \\
\hline acs $1 / 2 / 6 / 4 / 5 / 9 / 7 / 11$ & ACS isogene knock out ${ }^{\ddagger}$ \\
\hline
\end{tabular}

C
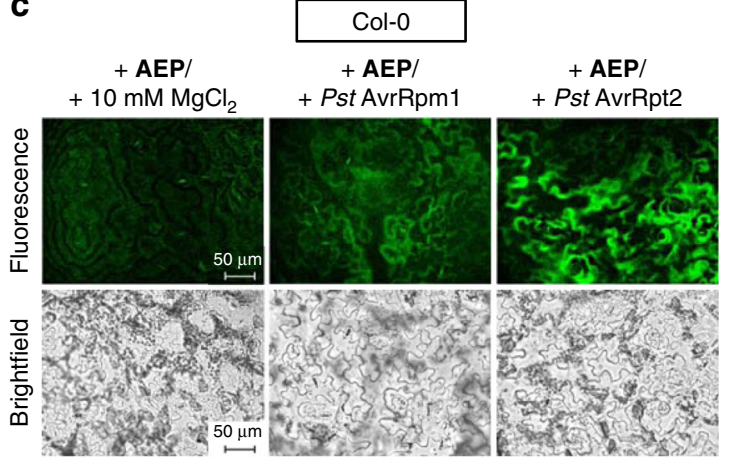

e

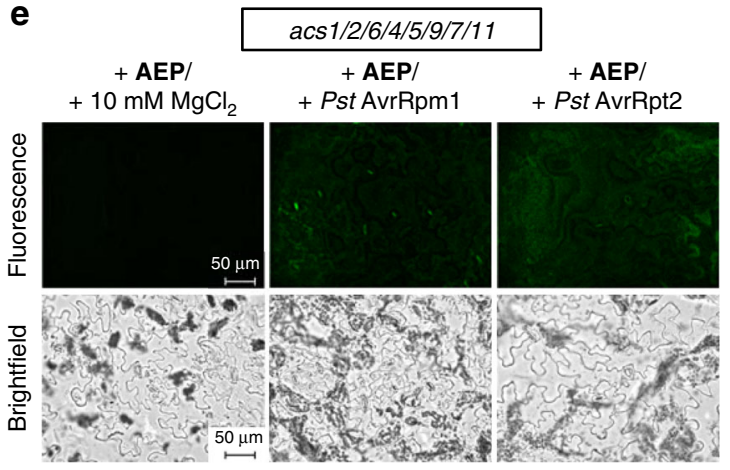

d

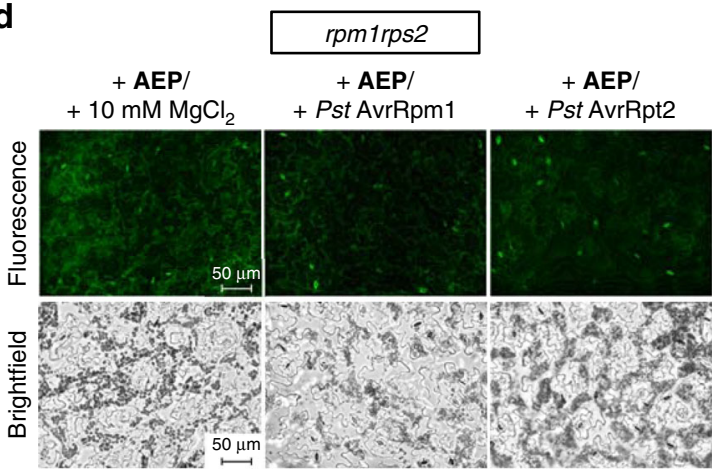

f

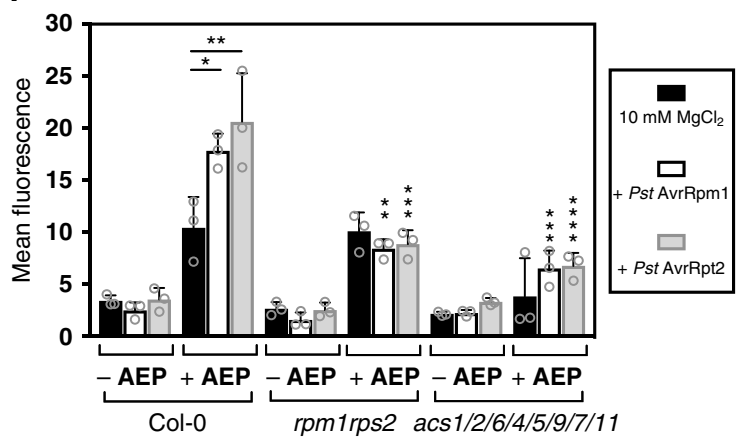

Fig. 9 Detection of ethylene produced as a result of effector-triggered immunity in $\boldsymbol{A}$. thaliana plants. a Depiction of the general pathway that leads to ETI response. $\mathbf{b}$ List of $A$. thaliana plants used in this experiment. Fluorescence and brightfield imaging ( $\times 40$ magnification) of epidermal peels applied with AEP $(100 \mu \mathrm{M})$ for wild-type Col-0 c, R-protein-deficient rpm1rps2 d, and the ethylene biosynthesis-defected acs 1/2/6/4/5/9/7/11 e. Additionally, supplementation studies were also performed using Pst AvrRpm1 or Pst AvrRpt2 (bacterial solution $\mathrm{OD}_{600}=0.02$ ), which was applied to all plants. f Summary of the measured fluorescence between the various conditions studied. Statistical analysis was performed using a one-way ANOVA with Tukey's multiple comparisons test. If not indicated, comparisons were made between the mutants and wild-type under the same treatment conditions. ${ }^{\star} P<0.03$, ${ }^{\star \star} P<0.002,{ }^{\star \star \star} P<0.0002,{ }^{\star \star \star \star} P<0.0001, \mathrm{~ns}=$ not significant. Error is represented as s.d. of three independent experiments. Source data are provided as a Source Data file.

With the advent of ArMs, a highly unique opportunity exists for the development of "artificial" enzyme biosensors, where metabolite detection can be driven via abiotic metal catalytic reactions. To the best of our knowledge, this study represents a leading example of an ArM being developed into an enzyme biosensor that has shown robust application with real world biological samples, such as fruits and leaves. Given its ease of preparation, there is the added benefit that this work can help pioneer the field of ArM biosensors, where different transition metals (e.g. palladium, rhodium, ruthenium, etc.) can be exploited for the chemoselective detection and quantification of other important biological metabolites.
Another significant aspect of this work is related to the development of the ethylene-sensing AEP probe, which has proven to be capable of detecting changes (both induced endogenously and exogenously) in biological concentrations of ethylene in various fruits and A. thaliana plants. Given the physiological importance of ethylene for plants, numerous studies have been conducted to understand ethylene-dependent pathways. However, one limitation of current detection methods (e.g. gas chromatography) is that typically only the average ethylene gas released into the surrounding environment can be measured. Without a proper bioimaging technique that can spatially detect ethylene on a cellular level, it is presently a challenge for scientists 
to determine the location and source of ethylene production. Despite some restrictions, this study has developed an ethylenesensing bioimaging technique shown to be capable of measuring ethylene in a spatiotemporal manner for cross-sections of various fruits and leaves.

\section{Methods}

General materials. Reagents and buffer components were purchased from SigmaAldrich, Fisher Scientific, Alfa Aesar, TCI, or Wako Chemicals without further purification. Human serum albumin (lyophilized powder, essentially fatty acid free) was purchased from Sigma-Aldrich (Prod\# A1887). All experiments dealing with air-sensitive and moisture-sensitive compounds were conducted under an atmosphere of dry nitrogen. Anhydrous solvents were used as received, which include THF (anhydrous; Fujifilm Wako Pure Chemical), dichloromethane (anhydrous; Fijifilm Wako Pure Chemical) and benzene (anhydrous; Fujifilm Wako Pure Chemical). TLC analyses (F-254) were performed with $60 \AA$ silica gel from Merck. ${ }^{1} \mathrm{H}$ and ${ }^{13} \mathrm{C}$ NMR spectra were measured on either a JEOL JNM-AL300 (300 MHz), JNM-ECZ400R/S1 $(400 \mathrm{MHz})$, or JNM-ECA500 $(500 \mathrm{MHz})$ instrument with the solvent peaks as internal standards: $\delta \mathrm{H} 0.00$ (tetramethylsilane) and $\delta \mathrm{C} 77.0$ for $\mathrm{CDCl}_{3}$. For chemical synthesis, high-resolution mass spectra (HRMS) were obtained on a Bruker MicroTOF-QIII spectrometer ${ }^{\circledR}$ by electron spray ionization time-of-flight (ESI-TOF-MS). Ultrapure water used for all experiments was obtained from a Milli-Q Advantage ${ }^{\oplus}$ A10 Water Purification System sold by Merck Millipore (Burlington, USA). In addition, Amicon ${ }^{\circledR}$ Ultra Centrifugal Filters (10 and $30 \mathrm{kDa}$ ) and Durapore PVDF $0.45 \mu \mathrm{m}^{\oplus}$ filters were also purchased from by Merck Millipore (Burlington, USA). All fruit and vegetable samples were purchased from a local grocery store. Previously published $A$. thaliana mutants were: rpm1rps2 (rpm1-3/rps2-101C) $)^{2}$, eto 1-162, acs1/2/6/4/5/9/7/11 ${ }^{60}$, and fls2/efr/ cerk $1^{66,70}$. The following bacterial strains were used in this study: $P$. syringae pv. tomato DC3000 transformed with pVSP61-AvrRpt2 (Pst AvrRpt2) and pVSP61AvrRpm1 (Pst AvrRpm1) ${ }^{75}$. Bacteria were propagated on LB media containing selection antibiotics and suspended with a $10 \mathrm{mM} \mathrm{MgCl}_{2}$ solution at a concentration of $\mathrm{OD}_{600}=1.0$.

Preparation of AEP. As previously described ${ }^{34}$, alb-Ru was prepared first by mixing $30 \mu \mathrm{M}$ of human serum albumin $(50 \mathrm{nmol}, 167 \mu \mathrm{l}$ from $300 \mu \mathrm{M}$ stock solution in $\left.\mathrm{H}_{2} \mathrm{O}\right)$ and $37 \mu \mathrm{M}$ of DEAC-Ru $(62 \mathrm{nmol}, 167 \mu \mathrm{l}$ from $370 \mu \mathrm{M}$ stock solution in dioxane). The total reaction volume was adjusted to $1670 \mu \mathrm{l}$ of $10 \%$ dioxane in PBS buffer $\mathrm{pH}$ 7.4. Following initiation by HSA addition, reaction mixtures were mildly mixed and incubated at $37^{\circ} \mathrm{C}$ for $1 \mathrm{~h}$. The reaction mixture was then concentrated and washed with PBS buffer $(3 \times)$ using Amicon ${ }^{\circledast}$ ultra centrifugal filters $(30 \mathrm{kDa})$. The concentrated alb-Ru solution was then diluted in $\mathrm{H}_{2} \mathrm{O}$ to $50 \mu \mathrm{l}$ to obtain a $1 \mathrm{mM}$ stock solution. To prepare the AEP solution, a mixture of $100 \mu \mathrm{M}$ of alb-Ru ( $50 \mathrm{nmol}, 50 \mu \mathrm{l}$ from $1 \mathrm{mM}$ stock solution in $\mathrm{H}_{2} \mathrm{O}$ ) and $500 \mu \mathrm{M}$ DABCYL quencher $(250 \mathrm{nmol}, 450 \mu \mathrm{l}$ from $555 \mu \mathrm{M}$ stock solution in 1:8 DMSO in $\mathrm{H}_{2} \mathrm{O}$ ) was made. Solutions were mildly mixed and incubated at $37^{\circ} \mathrm{C}$ for $5 \mathrm{~min}$. The reaction mixture was then concentrated and washed with $\mathrm{H}_{2} \mathrm{O}(3 \times)$ using Amicon ${ }^{\circledast}$ ultra centrifugal filters $(30 \mathrm{kDa})$. The concentrated AEP solution was then diluted in $\mathrm{H}_{2} \mathrm{O}$ to $500 \mu \mathrm{l}$ to obtain a $100 \mu \mathrm{M}$ stock solution. Following preparation, AEP was characterized via fluorescence spectroscopy and CD.

Protein complex fluorescence. For the protein complexes used in this study (alb-Ru, AEP), fluorescence was determined via spectrofluorometric analysis using a JASCO FP-6500 Spectrofluorometer equipped with a JASCO FMP-963 microplate reader. For sample preparations, both alb-Ru and AEP were adjusted to a concentration of $10 \mu \mathrm{M}$ in $\mathrm{H}_{2} \mathrm{O}$. Samples were then aliquoted (100 $\mu \mathrm{l}$ ) into 96-well microtiter plates, and monitored at $\lambda_{\mathrm{EX}}=420 \mathrm{~nm} / \lambda_{\mathrm{EM}}=463 \mathrm{~nm}$. All measurements were taken in triplicate from distinct samples.

CD experiments. In order to identify major structural changes to the various protein complexes used in this study (alb-Ru, AEP), CD analysis was done. $\mathrm{CD}$ spectra were measured on a J-1500 CD spectrometer (JASCO, Tokyo, Japan) using a $0.1 \mathrm{~cm}$ cell. A solution of $10 \%$ dioxane in water was used as a blank and automatically subtracted from the samples during scanning. Data was recorded from 200 to $250 \mathrm{~nm}$ with a scan speed of $100 \mathrm{~nm} / \mathrm{min}$. The concentration of each protein complex was maintained at $2.3 \mu \mathrm{M}$.

Ethylene concentration by NMR analysis. As an internal standard, a $100 \mathrm{mM}$ stock solution of 1,3-propanediol in THF-d8 was first prepared. For the $100 \%$ THF condition, an NMR tube was filled with $60 \mu \mathrm{l}$ of the internal standard solution and $540 \mu \mathrm{l}$ of THF-d8. For the $25 \% \mathrm{THF} / \mathrm{H}_{2} \mathrm{O}$ condition, an NMR tube was filled with $60 \mu \mathrm{l}$ of the internal standard solution, $90 \mu \mathrm{l}$ of THF-d8, and $450 \mu \mathrm{l}$ of $\mathrm{D}_{2} \mathrm{O}$. For the $10 \% \mathrm{THF} / \mathrm{H}_{2} \mathrm{O}$ condition, an NMR tube was filled with $60 \mu \mathrm{l}$ of the internal standard solution and $540 \mu \mathrm{l}$ of $\mathrm{D}_{2} \mathrm{O}$. Tubes were gastight sealed, before $1 \mathrm{ml}$ of ethylene gas was injected into each NMR tube. Following an incubation period of $5 \mathrm{~min}$, the ${ }^{1} \mathrm{H}$ NMR spectra was obtained. Ethylene concentrations in solution were determined through integration and comparison with the internal standard.
Dose-dependent reactivity of RuQ with ethylene gas. For dose-dependent reactivity experiments, a $100 \mu \mathrm{M}$ solution of $\mathbf{R u Q}$ in solvent (either $100 \%$ THF or $10 \%$ THF in $\mathrm{H}_{2} \mathrm{O}$ ) was prepared in a septum-sealed cuvette. Before injection, a measurement was recorded $(t=0 \mathrm{~min})$. A specific volume of ethylene gas (1000, $500,250,100,50 \mu \mathrm{l})$ was then quickly bubbled into solution. The cuvette was incubated for $1 \mathrm{~min}$ before another measurement was recorded $(t=1 \mathrm{~min})$. Fluorescence measurements were made using a JASCO FP-6500 spectrofluorometer $\left(\lambda_{\mathrm{EX}}=420 \mathrm{~nm} / \lambda_{\mathrm{EM}}=463 \mathrm{~nm}\right)$. All reactivity studies were performed in duplicate from distinct samples.

Time-based reactivity of AEP with ethylene gas. For time-based monitoring of AEP reactivity, water $(1425 \mu \mathrm{l})$ was added to a septum-sealed cuvette and purged with a balloon of ethylene gas. A solution of AEP $(75 \mu$ from $2 \mathrm{mM}$ stock solution in $\mathrm{H}_{2} \mathrm{O}$ ) was then added via syringe. The cuvette was then incubated at r.t. and monitored at set time intervals $(10,30,60,120$, and $180 \mathrm{~min})$. Fluorescence measurements were made using a JASCO FP-6500 spectrofluorometer $\left(\lambda_{\mathrm{Ex}}=\right.$ $420 \mathrm{~nm} / \lambda_{\mathrm{EM}}=463 \mathrm{~nm}$ ). All reactivity studies were performed in triplicate from distinct samples.

AEP-selectivity assay. To investigate AEP reactivity with various terpenes, water (1275 $\mu$ l) was first added to a cuvette, followed by a solution of AEP ( $75 \mu$ from $2 \mathrm{mM}$ stock solution in $\mathrm{H}_{2} \mathrm{O}$ ) and various terpenes $(150 \mu \mathrm{l}$ from $60 \mathrm{mM}$ stock solution in THF). The cuvette was then incubated at r.t. and monitored at $60 \mathrm{~min}$. Fluorescence measurements were made using a JASCO FP-6500 spectrofluorometer $\left(\lambda_{\mathrm{EX}}=420 \mathrm{~nm} / \lambda_{\mathrm{EM}}=463 \mathrm{~nm}\right)$. All reactivity studies were performed in triplicate from distinct samples.

Spatial imaging with kiwifruit. Unripe and ripening kiwifruit were obtained from the local supermarket. Sections of kiwifruit were prepared using a kitchen knife at rough dimensions of about $2.0 \mathrm{~cm} \times 4.5 \mathrm{~cm}$, where the aim was to obtain slices that included the outer pericarp, locules, and the columella of the fruit. To monitor ethylene production, $170 \mu \mathrm{l}$ of AEP $(400 \mu \mathrm{M}$ solution) was first applied to the center of a $10 \mathrm{~cm}$ Petri dish. Samples were then gently placed on top of the AEP solution to ensure even exposure. Samples were left to incubate at r.t., where imaging was carried at certain time intervals $(1,24 \mathrm{~h})$ using a Keyence BZ-X710 All-in-one Fluorescence Microscope ${ }^{\circledR}$ equipped with a ET-EBFP2/Coumarin/ Attenuated DAPI Filter Set Cat\#49021 (Chroma Technology Corp., Vermont, USA). Brightfield images (color) were obtained at a $1 / 25 \mathrm{~s}$ exposure setting and fluorescent images were obtained at a 1/3.5 s exposure setting. Multiple images were obtained at $\times 4$ magnification, where image stitching and analysis was performed using BZ-X Analyzer software (Keyence, Japan).

Spatial imaging with Asian pears. Asian pears were obtained from the local supermarket. Sections of flesh (hypanthium tissue) were prepared using a kitchen slicer, at rough dimensions of about $4.8 \mathrm{~cm} \times 4.8 \mathrm{~cm}$. Samples were then positioned in the center of a $10 \mathrm{~cm}$ Petri dish, where cotton balls were placed on the sample surface and allowed to incubate for $5 \mathrm{~h}$ at r.t. Four of the five cotton balls are soaked with water, while the remaining cotton ball is soaked with either $10 \mathrm{mM}$ ACC or $10 \mathrm{mM}$ PZA. To monitor ethylene production, $300 \mu \mathrm{l}$ of AEP $(400 \mu \mathrm{M}$ solution) was first applied to the center of another $10 \mathrm{~cm}$ Petri dish. Samples were then gently placed on top of the AEP solution to ensure even exposure. Samples were left to incubate at r.t., where imaging was carried out after $18 \mathrm{~h}$ using a Keyence BZ-X710 All-in-one Fluorescence Microscope ${ }^{\circledast}$ equipped with a ETEBFP2/Coumarin/Attenuated DAPI Filter Set Cat\#49021 (Chroma Technology Corp., Vermont, USA). Brightfield images (color) were obtained at a $1 / 25 \mathrm{~s}$ exposure setting and fluorescent images were obtained at a $1 / 3.5 \mathrm{~s}$ exposure setting. Multiple images were obtained at $\times 4$ magnification, where image stitching and analysis was performed using BZ-X Analyzer software (Keyence, Japan).

Time-dependent imaging with fruits. Apples (Golden Delicious, Jazz, and Fuji cultivars), Asian pears, kiwifruit, and Muscat grapes, carrots, and red bell peppers were obtained from the local supermarket. Samples of tissue (small rectangles of $\sim 10 \mathrm{~mm} \times 8 \mathrm{~mm}$ ) were prepared using a kitchen slicer and knife. Samples were then incubated in either water, ACC (1 or $10 \mathrm{mM})$, or PZA $(1$ or $10 \mathrm{mM})$ for $3 \mathrm{~h}$ at r.t. To monitor ethylene production, $10 \mu \mathrm{l}$ of AEP ( $400 \mu \mathrm{M}$ solution) was first applied to the center of individual wells of a chamber slide (ibidi $\mu$-Slide eight-well chamber slides). Samples were then gently placed on top of the AEP solution to ensure even exposure. Samples were left to incubate at r.t., where imaging was carried at certain time intervals $(0,1,2,4,8,20 \mathrm{~h})$ using a Keyence BZ-X710 All-inone Fluorescence Microscope ${ }^{\circledast}$ equipped with a ET-EBFP2/Coumarin/Attenuated DAPI Filter Set Cat\#49021 (Chroma Technology Corp., Vermont, USA). Brightfield images (color) were obtained at a 1/80 s exposure setting and fluorescent images were obtained at a $1 / 6 \mathrm{~s}$ exposure setting. Multiple images were obtained at $\times 4$ magnification, where image stitching and analysis was performed using BZ-X Analyzer software (Keyence, Japan).

Imaging with $\boldsymbol{A}$. thaliana plants. A. thaliana plants were grown on soil at $23^{\circ} \mathrm{C}$ and a light intensity of $85 \mu \mathrm{mol} \mathrm{m}{ }^{-2} \mathrm{~s}^{-1}$. A $10 \mathrm{~h}$ light and $14 \mathrm{~h}$ dark photoperiod 
was applied. To monitor ethylene production, leaves were first harvested from 4 to 6 weeks old plants. Using forceps, clear epidermal peels were then removed from leaves and placed into 96-well clear bottom plates. Following the addition of $\mathrm{H}_{2} \mathrm{O}$ $(100 \mu \mathrm{l})$, the samples were then incubated for $12 \mathrm{~h}$ at r.t. to cancel wound stress. Depending on the conditions under study, various solutions were added as necessary: $1 \mathrm{mM}$ of ACC $\left(2 \mu \mathrm{l}\right.$ of $55 \mathrm{mM}$ stock solution in $\left.\mathrm{H}_{2} \mathrm{O}\right) ; 4.8 \mu \mathrm{M}$ of flg22 or elf18 $\left(5 \mu \mathrm{l}\right.$ of $100 \mu \mathrm{M}$ stock solution in $\left.\mathrm{H}_{2} \mathrm{O}\right)$; or $\mathrm{OD}_{600}=0.02$ solution of Pseudomonas bacteria $\left(2 \mu \mathrm{l}\right.$ of $\mathrm{OD}_{600}=1.0$ stock solution). For studies involved with ACC and Pseudomonas bacteria, an incubation period of $12 \mathrm{~h}$ at r.t. was applied. For studies involved with PAMPs, an incubation period of $6 \mathrm{~h}$ at r.t. was instead applied. The solutions were then completely removed from the epidermal peel samples, followed by the addition of $100 \mu \mathrm{M}$ AEP ( $50 \mu \mathrm{l}$ stock solution). After 30 min, solutions were completely removed, washed with $\mathrm{H}_{2} \mathrm{O}$, and then imaged on a Keyence BZ-X710 All-in-one Fluorescence Microscope ${ }^{\circledR}$ equipped with a ETEBFP2/Coumarin/Attenuated DAPI Filter Set Cat\#49021 (Chroma Technology Corp., Vermont, USA). Images were obtained at $\times 20$ and $\times 40$ magnification, where brightfield images (monochrome) were obtained at a 1/400 s exposure setting and fluorescent images were obtained at a 1/30 s exposure setting. Analysis was performed using BZ-X Analyzer software (Keyence, Japan).

Reporting summary. Further information on research design is available in the Nature Research Reporting Summary linked to this article.

\section{Data availability}

All data supporting the findings of this study are available with the article, and can also be obtained from the corresponding author upon reasonable request. The source data underlying Figs. 2b-g; 3b; 4e; 5b-e; 6a-b, d-e, g-h; 7e; 8f; 9f, and Supplementary Figs. 42a, b, d, e are provided as a Source Data file.

Received: 7 June 2019; Accepted: 20 November 2019; Published online: 17 December 2019

\section{References}

1. Bari, R. \& Jones, J. D. G. Role of plant hormones in plant defence responses. Plant Mol. Biol. 69, 473-488 (2009).

2. Iqbal, N. et al. Ethylene role in plant growth, development and senescence: interaction with other phytohormones. Front. Plant Sci. 8, 475 (2017).

3. McMurchie, E. J., McGlasson, W. B. \& Eaks, I. L. Treatment of fruit with propylene gives information about the biogenesis of ethylene. Nature 237, 235-236 (1972).

4. Alexander, L. \& Grierson, D. Ethylene biosynthesis and action in tomato: a model for climacteric fruit ripening. J. Exp. Bot. 53, 2039-2055 (2002).

5. Lelièvre, J.-M. et al. Ethylene and fruit ripening. Physiol. Plant 101, 727-739 (1997).

6. Barry, C. S., Llop-Tous, M. I. \& Grierson, D. The regulation of 1aminocyclopropane-1-carboxylic acid synthase gene expression during the transition from system-1 to system-2 ethylene synthesis in tomato. Plant Physiol. 123, 979-986 (2000).

7. Yokotani, N. et al. Ripening-associated ethylene biosynthesis in tomato fruit is autocatalytically and developmentally regulated. J. Exp. Bot. 60, 3433-3442 (2009).

8. Lipe, J. A. \& Morgan, P. W. Ethylene, a regulator of young fruit abscission. Plant Physiol. 51, 949-953 (1973).

9. Brown, K. M. Ethylene and abscission. Physiol. Plant 100, 567-576 (1997).

10. Burg, S. P. \& Burg, E. A. Role of ethylene in fruit ripening. Plant Physiol. 37, 179-189 (1962).

11. Saltveit, M. E. Effect of ethylene on quality of fresh fruits and vegetables. Postharvest Biol. Technol. 15, 279-292 (1999).

12. Arslanov, D. et al. Current methods for detecting ethylene in plants. Ann. Bot. 111, 347-360 (2012).

13. Fernandez-Moreno, J.-P. \& Stepanova, A. N. Monitoring ethylene in plants: genetically encoded reporters and biosensors. Small Methods 1900260, https:// doi.org/10.1002/smtd.201900260 (2019).

14. Stepanova, A. N., Yun, J., Likhacheva, A. V. \& Alonso, J. M. Multilevel interactions between ethylene and auxin in Arabidopsis roots. Plant Cell 19, 2169-2185 (2007).

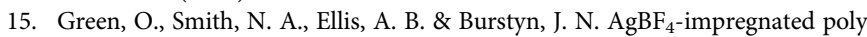
(vinyl phenyl ketone): an ethylene sensing film. J. Am. Chem. Soc. 126, 5952-5953 (2004).

16. Esser, B. \& Swager, T. M. Detection of ethylene cas by fluorescence turn-on of a conjugated polymer. Angew. Chem. Int. Ed. Engl. 49, 8872-8875 (2010).

17. Hitomi, Y., Nagai, T. \& Kodera, M. A silver complex with an N,S,Smacrocyclic ligand bearing an anthracene pendant arm for optical ethylene monitoring. Chem Comm 48, 10392-10394 (2012).
18. Toussaint, S. N. W., Calkins, R. T., Lee, S. \& Michel, B. W. Olefin metathesisbased fluorescent probes for the selective detection of ethylene in live cells. J. Am. Chem. Soc. 140, 13151-13155 (2018).

19. Sun, M. et al. Rapid and visual detection and quantitation of ethylene released from ripening fruits: the new use of Grubbs catalyst. J. Agric. Food Chem. 67, 507-513 (2019).

20. Noctor, G. et al. Glutathione in plants: an integrated overview. Plant Cell Environ. 35, 454-484 (2012).

21. Hyster, T. K., Knorr, L., Ward, T. R. \& Rovis, T. Biotinylated Rh(III) complexes in engineered streptavidin for accelerated asymmetric $\mathrm{C}-\mathrm{H}$ activation. Science 338, 500-503 (2012).

22. Jeschek, M. et al. Directed evolution of artificial metalloenzymes for in vivo metathesis. Nature 537, 661-665 (2016).

23. Köhler, V. et al. Synthetic cascades are enabled by combining biocatalysts with artificial metalloenzymes. Nat. Chem. 5, 93-99 (2012).

24. Okamoto, Y. et al. A cell-penetrating artificial metalloenzyme regulates a gene switch in a designer mammalian cell. Nat. Commun. 9, 1943 (2018).

25. Heinisch, T. et al. E. coli surface display of streptavidin for directed evolution of an allylic deallylase. Chem. Sci. 9, 5383-5388 (2018).

26. Szponarski, M., Schwizer, F., Ward, T. R. \& Gademann, K. On-cell catalysis by surface engineering of live cells with an artificial metalloenzyme. Commun. Chem. 1, 84 (2018).

27. Yang, H. et al. Evolving artificial metalloenzymes via random mutagenesis. Nat. Chem. 10, 318-324 (2018).

28. Srivastava, P., Yang, H., Ellis-Guardiola, K. \& Lewis, J. C. Engineering a dirhodium artificial metalloenzyme for selective olefin cyclopropanation. Nat. Commun. 6, 7789 (2015).

29. Key, H. M., Dydio, P., Clark, D. S. \& Hartwig, J. F. Abiological catalysis by artificial haem proteins containing noble metals in place of iron. Nature 534, 534-537 (2016).

30. Hayashi, T. et al. Capture and characterization of a reactive haem-carbenoid complex in an artificial metalloenzyme. Nat. Catal. 1, 578-584 (2018).

31. Raines, D. J. et al. Redox-switchable siderophore anchor enables reversible artificial metalloenzyme assembly. Nat. Catal. 1, 680-688 (2018).

32. Ghattas, W. et al. Receptor-based artificial metalloenzymes on living human cells. J. Am. Chem. Soc. 140, 8756-8762 (2018).

33. Grimm, A. R. et al. A whole cell $E$. coli display platform for artificial metalloenzymes: poly(phenylacetylene) production with a rhodium-nitrobindin metalloprotein. ACS Catal. 8, 2611-2614 (2018).

34. Eda, S. et al. Biocompatibility and therapeutic potential of glycosylated albumin artificial metalloenzymes. Nat. Catal. 2, 780-792 (2019).

35. An, F. F. \& Zhang, X. H. Strategies for preparing albumin-based nanoparticles for multifunctional bioimaging and drug delivery. Theranostics 7, 3667-3689 (2017).

36. Malamud, D. \& Drysdale, J. W. Isoelectric points of proteins: a table. Anal. Biochem. 86, 620-647 (1978).

37. Ogura, A. et al. Visualizing trimming dependence of biodistribution and kinetics with homo- and heterogeneous N-glycoclusters on fluorescent albumin. Sci. Rep. 6, 21797 (2016)

38. Ogura, A. et al. Glycan multivalency effects toward albumin enable N-glycandependent tumor targeting. Bioorg. Med. Chem. Lett. 26, 2251-2254 (2016).

39. Latypova, L. et al. Sequential double "clicks" toward structurally well-defined heterogeneous N-glycoclusters: the importance of cluster heterogeneity on pattern recognition in vivo. Adv. Sci. 4, 1600394 (2017).

40. Tsubokura, K. et al. In vivo gold complex catalysis within live mice. Angew. Chem. Int. Ed. Engl. 56, 3579-3584 (2017).

41. Ogura, A. et al. A viable strategy for screening the effects of glycan heterogeneity on target organ adhesion and biodistribution in live mice. ChemComm 54, 8693-8696 (2018).

42. He, G. et al. A coumarin-based fluorescence resonance energy transfer probe targeting matrix metalloproteinase- 2 for the detection of cervical cancer. Int. J. Mol. Med. 39, 1571-1579 (2017).

43. Webster, C. E. Computational insights into degenerate ethylene exchange with a Grubbs-type catalyst. J. Am. Chem. Soc. 129, 7490-7491 (2007).

44. Jones, J. D. G. \& Dangl, J. L. The plant immune system. Nature 444, 323-329 (2006).

45. Boller, T. \& Kende, H. Regulation of wound ethylene synthesis in plants. Nature 286, 259-260 (1980).

46. Ikoma, Y., Yano, M., Xu, Z. C. \& Ogawa, K. Isolation of a cDNA encoding active protein for kiwifruit ACC synthase and its specific expression in the outer pericarp. J. Jpn. Soc. Hort. Sci. 68, 286-288 (1999).

47. Ikoma, Y., Yano, M., Chuan Xu, Z. \& Ogawa, K. Reduction in ethylene synthesis in parthenocarpic Actinidia deliciosa fruit induced by $\mathrm{N}$-(2-chloro4-pyridyl)-N'-phenylurea. Postharvest Biol. Technol. 13, 121-129 (1998).

48. Kende, H. Enzymes of ethylene biosynthesis. Plant Physiol. 91, 1-4 (1989).

49. Acaster, M. A. \& Kende, H. Properties and partial purification of 1aminocyclopropane-1-carboxylate synthase. Plant Physiol. 72, 139-145 (1983). 
50. Van de Poel, B. et al. Targeted systems biology profiling of tomato fruit reveals coordination of the Yang cycle and a distinct regulation of ethylene biosynthesis during postclimacteric ripening. Plant Physiol. 160, 1498-1514 (2012).

51. Houben, M. \& Van de Poel, B. 1-Aminocyclopropane-1-carboxylic acid oxidase (ACO): the enzyme that makes the plant hormone ethylene. Front. Plant Sci. 10, 695 (2019).

52. Sun, X. et al. Pyrazinamide and derivatives block ethylene biosynthesis by inhibiting ACC oxidase. Nat. Commun. 8, 15758 (2017).

53. Blankenship, S. M. \& Unrath, C. R. Internal ethylene levels and maturity of delicious and golden delicious apples destined for prompt consumption. J. Am. Soc. Hortic. Sci. 113, 88-91 (1988).

54. Chu, C. L. Use of internal ethylene concentration as a maturity index of eleven apple cultivars. Acta Hortic. 157, 129-134 (1985).

55. Jobling, J. J. \& McGlasson, W. B. A comparison of ethylene production, maturity and controlled atmosphere storage life of Gala, Fuji and Lady Williams apples (Malus domestica, Borkh.). Postharvest Biol. Technol. 6, 209-218 (1995).

56. Hyodo, H. \& Fukasawa, R. Ethylene production in kiwifruit. J. Jpn. Soc. Hortic. Sci. 54, 209-215 (1985).

57. Itai, A., Tanabe, K. \& Tamura, F. Mechanism of ethylene production during fruit ripening in Asian pears. Acta Hortic. 587, 497-504 (2002).

58. Chervin, C. et al. Ethylene seems required for the berry development and ripening in grape, a non-climacteric fruit. Plant Sci. 167, 1301-1305 (2004).

59. Dal Ri, A. et al. Ethylene production during grape berry development and expression of genes involved in ethylene biosynthesis and response. Acta Hortic. 884, 73-80 (2010).

60. Tsuchisaka, A. et al. A combinatorial interplay among the 1aminocyclopropane-1-carboxylate isoforms regulates ethylene biosynthesis in Arabidopsis thaliana. Genetics 183, 979-1003 (2009).

61. Guan, R. et al. Multilayered regulation of ethylene induction plays a positive role in Arabidopsis resistance against Pseudomonas syringae. Plant Physiol. 169, 299-312 (2015).

62. Guzmán, P. \& Ecker, J. R. Exploiting the triple response of Arabidopsis to identify ethylene-related mutants. Plant Cell 2, 513-523 (1990).

63. Yoshida, H. et al. Arabidopsis ETO1 specifically interacts with and negatively regulates type 2 1-aminocyclopropane-1-carboxylate synthases. BMC Plant Biol. 5, 14 (2005).

64. Wu, S., Shan, L. \& He, P. Microbial signature-triggered plant defense responses and early signaling mechanisms. Plant Sci. 228, 118-126 (2014).

65. Boutrot, F. et al. Direct transcriptional control of the Arabidopsis immune receptor FLS2 by the ethylene-dependent transcription factors EIN3 and EIL1. Proc. Natl Acad. Sci. USA 107, 14502-14507 (2010).

66. Gomez-Gomez, L. \& Boller, T. FLS2: An LRR receptor-like kinase involved in the perception of the bacterial elicitor flagellin in Arabidopsis. Mol. Cell 5, 1003-1011 (2000).

67. Felix, G., Duran, J. D., Volko, S. \& Boller, T. Plants have a sensitive perception system for the most conserved domain of bacterial flagellin. Plant J. 18 265-276 (1999).

68. Asai, T. et al. MAP kinase signalling cascade in Arabidopsis innate immunity. Nature 415, 977-983 (2002).

69. Kunze, G. et al. The $\mathrm{N}$ terminus of bacterial elongation factor Tu elicits innate immunity in Arabidopsis plants. Plant Cell 16, 3496-3507 (2004).

70. Zipfel, C. et al. Perception of the bacterial PAMP EF-Tu by the receptor EFR restricts Agrobacterium-mediated transformation. Cell 125, 749-760 (2006).

71. Kim, M. G. et al. Two Pseudomonas syringae type III effectors inhibit RIN4regulated basal defense in Arabidopsis. Cell 121, 749-759 (2005).

72. Mackey, D., Holt, B. F. III, Wiig, A. \& Dangl, J. L. RIN4 interacts with Pseudomonas syringae type III effector molecules and is required for RPM1mediated resistance in Arabidopsis. Cell 108, 743-754 (2002).

73. Mackey, D. et al. Arabidopsis RIN4 is a target of the type III virulence effector AvrRpt2 and modulates RPS2-mediated resistance. Cell 112, 379-389 (2003).
74. Axtell, M. J. \& Staskawicz, B. J. Initiation of RPS2-specified disease resistance in Arabidopsis is coupled to the AvrRpt2-directed elimination of RIN4. Cell 112, 369-377 (2003).

75. Kunkel, B. N. et al. RPS2, an Arabidopsis disease resistance locus specifying recognition of Pseudomonas syringae strains expressing the avirulence gene avrRpt2. Plant Cell 5, 865-875 (1993).

\section{Acknowledgements}

Kind acknowledgement is given to GlyTech Inc. for supporting this research. CD spectral measurements were acquired by the Molecular Structure Characterization Unit, RIKEN Center for Sustainable Resource Science (CSRS). This work was financially supported by the MEXT/JSPS KAKENHI Grant Numbers JP16H03287, JP18K19154, JP15H05843 (to K.T.), JP16H06186 (to Y.K.), JP15H05959 (to K. S.), and JP18K14347 (to K.V.). This work was also supported by a RIKEN Incentive Research Project grant to I.N. and Y.K., as well as the Russian Government Program for Competitive Growth (granted to Kazan Federal University).

\section{Author contributions}

Preparation of reagents was done by S.E., K.V., and I.N. Preparation of plant and bacterial strains were carried out by Y.K. Fruit-imaging studies were carried out by K.V., while plant-imaging studies were done by S.E. and T.W. Reactivity and spectroscopic studies were performed by K.V. and S.E. The manuscript was written by K.V. and K.T., and checked by S.Y. and K.S. This research was directed and supervised by K.T.

\section{Competing interests}

The authors declare no competing interests.

\section{Additional information}

Supplementary information is available for this paper at https://doi.org/10.1038/s41467 019-13758-2.

Correspondence and requests for materials should be addressed to K.T.

Peer review information Nature Communications thanks Bram Van de Poel and the other, anonymous, reviewer(s) for their contribution to the peer review of this work. Peer reviewer reports are available.

Reprints and permission information is available at http://www.nature.com/reprints

Publisher's note Springer Nature remains neutral with regard to jurisdictional claims in published maps and institutional affiliations.

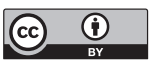

Open Access This article is licensed under a Creative Commons Attribution 4.0 International License, which permits use, sharing adaptation, distribution and reproduction in any medium or format, as long as you give appropriate credit to the original author(s) and the source, provide a link to the Creative Commons license, and indicate if changes were made. The images or other third party material in this article are included in the article's Creative Commons license, unless indicated otherwise in a credit line to the material. If material is not included in the article's Creative Commons license and your intended use is not permitted by statutory regulation or exceeds the permitted use, you will need to obtain permission directly from the copyright holder. To view a copy of this license, visit http://creativecommons.org/ licenses/by/4.0/.

(c) The Author(s) 2019 